\title{
PROPAGATION OF UNCERTAINTY BY SAMPLING ON CONFIDENCE BOUNDARIES
}

\author{
Jan Peter Hessling ${ }^{1, *}$ E Thomas Svensson ${ }^{2}$ \\ ${ }^{1}$ SP Technical Research Institute of Sweden, Measurement Technology, Box 857, SE-50115 Borås, \\ Sweden \\ ${ }^{2}$ SP Technical Research Institute of Sweden, Building Technology and Mechanics, Box 857, SE- \\ 50115 Borås, Sweden
}

Original Manuscript Submitted: 08/11/2011; Final Draft Received: 04/26/2012

\begin{abstract}
A new class of methods for propagation of uncertainty through complex models nonlinear-in-parameters is proposed. It is derived from a recent idea of propagating covariance within the unscented Kalman filter. The nonlinearity could be due to a pole-zero parametrization of a dynamic model in the Laplace domain, finite element model (FEM) or other large computer models, models of mechanical fatigue etc. Two approximate methods of this class are evaluated against Monte Carlo simulations and compared to the application of the Gauss approximation formula. Three elementary static models illustrate pros and cons of the methods, while one dynamic model provides a realistic simple example of its use.
\end{abstract}

KEY WORDS: unscented, deterministic, Kalman, sigma points, nonlinear, nondegenerate, covariance

\section{INTRODUCTION}

The variability or uncertainty of modeling is an important indicator of quality in many fields of science and engineering. Models are also used to evaluate the measurement uncertainty [1]. Typically, the uncertainties and correlations of parameters are more or less known or estimated from experiments, assumptions, or first principles. This information should then be propagated to the result of modeling. In this propagation there is no statistical evaluation, as the basic statistical information is already encoded in the parametric uncertainty. The widely spread approach to propagate the uncertainty $[1,2]$ is based on linearization and propagation of variances (LIN), according to the so-called "Gauss approximation formula." The resulting variance of the model is often expanded to a confidence interval by assignment of a coverage factor. Two important approximations are involved in LIN, namely (i) the model is linearized within the interval of interest and (ii) the statistical properties of the sources of uncertainty beyond their first and second statistical moments are neglected. The second approximation is usually unavoidable due to limited knowledge. More statistical details can be propagated with random sampling, or Monte Carlo simulations (MC) [3]. For the targeted complex models, none of these methods is satisfactory: The large computational burden of MC distinctively prohibits its application. Linearization is effective but may result in large errors.

Ever since their first utilization for propagation of uncertainty, the basic ideas of model approximations as in LIN, and statistical sampling [4] as in MC, have been refined. In polynomial chaos expansions (PCE) [5] the random fields of interest are approximated by expanding them in truncated series of random so-called polynomial chaos. Inserting these expansions into the original model, the governing equations are derived, which determine the unknown deterministic expansion coefficients [6]. Since the governing equations differ from the model equation, the PCE technique is invasive. Thus, for models with limited accessibility, PCE may not be applicable. Stratified sampling techniques, such as latin hypercube sampling (LHS), are used to improve the generation of random samples to allow for smaller sets giving higher computational efficiency. In LHS, a deterministic rule is used to obtain a complete set of stratas, i.e., disjoint subspaces $\epsilon_{p}$ which enclose the entire probability space $Q$ of all parameters, $\cup_{p} \epsilon_{p}=Q, \epsilon_{p} \cap \epsilon_{q}=\oslash, p \neq q$.

\footnotetext{
${ }^{*}$ Correspond to Jan Peter Hessling, E-mail: peter.hessling@sp.se, URL: http://www.sp.se/
}

DOI: $10.1615 /$ Int.J.UncertaintyQuantification.2012004275 
A given number of samples are then randomly drawn from each subspace $\epsilon_{p}$. In stochastic collocation (SCL) [7] a particular grid of so-called collocation points are used to evaluate integral statistics such as statistical moments by means of quadrature rules [8]. In contrast to sample points, the collocation points have no statistical meaning. Response surface methodology (RSM) [9] utilizes model approximations as well as statistical sampling. Collocation points are used in RSM to determine approximate simple surrogate models. This model is then sampled randomly, usually densely as in conventional "brute force" $\mathrm{MC}$, even though stratified techniques are perfectly allowed. The two-stage techniques of SCL and RSM are similar but different from our approach: Collocation points will not be used here to determine any model approximation to be densely sampled (RSM) or integrated (SCL), but rather to determine a minimal secondary set of points. The confidence interval of any quantity related to the modeling result is then obtained directly by sampling the model at these points, without any model approximation. Our method thus differs from all established methods for uncertainty propagation, LIN, MC, PCE, LHS, SCL, and RSM. The targets for our methods are the most complex uncertain models which can only be evaluated, but not analyzed as required in PCE. All proposed methods must thus be noninvasive. Our primary goal is to maximize computational efficiency by minimizing the number of model evalutions, but still account for nonlinear propagation of uncertainty.

An alternative to deterministic sampling is used in the "unscented Kalman filter" (UKF) [10-12]. The novel class of methods for estimating confidence intervals to be presented is related to the UKF, but also a recently proposed approach by one of the authors [13]. The latter method is here generalized from essentially one to any number of uncertain parameters and brought into the large perspective of deterministic sampling suggested by the UKF: Two "confidence boundaries" are first estimated. These are uniquely defined as the two disjoint sets of points in parameter space mapped to the desired confidence interval limits of the model result. Each estimated boundary is then sampled once to give two "lambda points" in total. Evaluating the model for these lambda points will by definition estimate the confidence interval limits of the model. In contrast to the LIN method [1], the expansion to the desired confidence level is made before evaluating the model. Errors of this expansion due to the nonlinear mapping through the model are then in principle eliminated. The coverage factors are also easier to estimate as they relate to the multivariate distribution of the parameters, instead of the output of a complex model. Non-linearities-in-parameters as well as dynamically induced correlations provide two major error sources of the coverage factors used in LIN, but none for the lambda points. The difficulty of our methods lies entirely in the estimation of the confidence boundaries. Two general approximate methods to estimate these boundaries will be discussed and illustrated. For a monotonic model of one uncertain parameter the confidence boundaries are just two points, the two given percentiles of the parameter. Since the confidence boundaries are then known, the confidence interval of the model can be determined exactly. Also in the multivariate case, the dependence on many details of the probability distribution of the parameters (in the interior) is often weak. For all linear models, the confidence boundaries can be determined exactly if the coverage factor is known. These facts will be extensively explored in this study.

The article is organized as follows: Before presenting our approach, its presumably less known background is given in a primer (Section 2). The confidence boundaries are introduced (Section 3) before they are sampled (Section 4), utilizing our novel Unscented Nondegenerate sampling with central Gradient approximation (UNG, Section 4.1) and Unscented Nondegenerate sampling using linear Regression (UNR, Section 4.2) methods. Their accuracy and applicability is then explored (Section 4.3). The determination of coverage factors provides a separate problem (Section 4.4). Dynamic models expressed by ordinary differential/difference equations require some generalization (Section 5). The result of using the methods are illustrated in three rudimentary static examples (Sections 6.1-6.3), and one realistic dynamic example (Section 6.4). Finally, a comparison to the seemingly closely related RSM (Section 7) precedes our conclusions (Section 8). For reference, the methods RSM (Appendix A) and linearization with regression (Appendix B) used for evaluating our methods are also briefly described.

\section{PRIMER}

The background and foundation for our approach is given in this primer, which includes the concept deterministic sampling (Section 2.1), aspects of nonlinear propagation (Section 2.2), and the formulation of the UKF (Section 2.3). 


\subsection{Deterministic Sampling}

Deterministic sampling is contrasted to random sampling techniques, or MC simulations where samples are drawn with a random generator. Both classes of methods sample statistical information to a finite number of sets of parameters collected into ensembles. The finite ensembles describe, or represent, the statistical information of the model. Deterministic sampling methods do not utilize random generators at all. A definite deterministic rule constructs samples from the available statistical information. Still, the deterministic ensemble is statistical [4], as it has a definite statistical meaning. As with all sampling, more or less information is unconditionally lost. Random sampling is constructed to yield a complete asymptotically correct representation of statistical information. Deterministic sampling is devised and optimized for a particular task of interest, like propagation of covariance [11]. Random sampling usually requires large ensembles (typically $10^{3}-10^{6}$ samples), while deterministic ensembles may, as in this work, contain as few as two(!) samples. The difference in efficiency between deterministic and random sampling may therefore be profound. The superior accuracy of random sampling makes it an ideal tool for verification, but at a very high computational cost. Deterministic sampling may employ any mathematical operation on the available statistical information. This freedom and lack of completeness makes the class of deterministic sampling techniques large and complex.

\subsection{Nonlinear Propagation of Uncertainty}

For nonlinear propagation of uncertainty, the asymmetry of the resulting probability distribution is important. It can be expressed as a lack of commutation between nonlinear propagation and statistical evaluation. The scent for a model $h(q): \Re^{n} \rightarrow \Re$ will be defined as

$$
\zeta=h_{c}(q)-h\left(q_{c}\right) .
$$

This is the commutator for evaluating the realized models $[h(\cdot)]$ and the center $\left({ }_{c}\right)$ of the confidence interval, or domain. The method for evaluating the center is left unspecified, as there are many alternatives. The second moment $\left\langle\left(q-q_{c}\right)^{2}\right\rangle$, where $\langle\cdot\rangle$ denotes statistical expectation, is for instance minimized by choosing $q_{c}=\langle q\rangle$. A nonlinear mapping $h$ of the mean $\langle q\rangle$ differs from the mean of the mapping, $h(\langle q\rangle) \neq\langle h(q)\rangle$. The associated scent $\langle h(q)\rangle-$ $h(\langle q\rangle)$ is completely neglected when the model is linearized. The lowest order approximation of the scent is in this case directly obtained with a Taylor expansion, $\zeta \approx \operatorname{Tr}[H(h) \operatorname{cov}(q)] / 2$, where $H(h)_{j k}=\partial^{2} h / \partial q_{k} \partial q_{j}$ is the Hessian matrix evaluated at $q=\langle q\rangle$. The scent is related to the skewness [14], $\gamma=\left\langle\delta q^{3}\right\rangle /\left\langle\delta q^{2}\right\rangle^{3 / 2}, \delta q=q-\langle q\rangle$, of a univariate probability distribution of $q$. The additional asymmetry caused by the nonlinearity of the model is measured with the "scent," but differently: Even if the distribution of a parameter $q$ has no skewness, a nonlinear model $h(q)$ will likely have a finite scent $\zeta=h_{c}(q)-h\left(q_{c}\right) \neq 0$, differing from its skewness $\left\langle[\delta h(q)]^{3}\right\rangle /\left\langle[\delta h(q)]^{2}\right\rangle^{3 / 2}, \delta h(q)=$ $h(q)-\langle h(q)\rangle$. Scent is to be distinguished from bias. The bias is a property of an estimator while the scent is a property of an uncertain model: In the definition (1) there is no reference to any estimator; the center $\left(\cdot{ }_{c}\right)$ is defined and not estimated. An estimator of a confidence interval (such as UNG/UNR to be proposed) is unbiased if it yields a finite scent equal to the true scent of the model. An unbiased estimator of $h_{c}$ may hence also be quoted "unscented" if the error of the estimated scent vanishes. A rather accurate estimation of scent is probably the most important property of the unscented Kalman filter (UKF), as well as the methods to be proposed in Section 4. We interpret this as the origin of the name unscented in the UKF. This context provides the motivation to introduce scent as a convenient concept when discussing nonlinear propagation of uncertainty.

The scent is generally larger and hence more important to evaluate than the nonlinear shift $\Delta h_{p}$ of the halfwidth $h_{p}$ of probabilistically symmetric confidence intervals. The half-width $h_{p}$ is complex to evaluate, but the lowest order nonlinear shift of the related variance is given by, $\Delta[\operatorname{var}(h)] \approx \sum_{j k l}\left\langle\delta q_{j} \delta q_{k} \delta q_{l}\right\rangle \partial h / \partial q_{j} H(h)_{k l}+$ $\sum_{j k l m}\left\langle\delta q_{j} \delta q_{k} \delta q_{l} \delta q_{m}\right\rangle \times\left[H(h)_{j k} H(h)_{l m} / 4+\partial h / \partial q_{j} \partial^{3} h / \partial q_{k} \partial q_{l} \partial q_{m} / 3\right]$. For symmetric probability distributions the first term vanishes. Ignoring the nonlinear shift of the coverage factor, the nonlinear correction $\Delta h_{p}$ is thus at least one but often two orders less than the scent $\zeta$.

\subsection{Propagation of Covariance in the Unscented Kalman Filter}

For optimal state estimation in the time domain, Kalman filtering has been extensively studied since it was first proposed about half a century ago [15]. For nonlinear systems, the standard Kalman filter was developed into the extended 
Kalman filter (EKF) based on Taylor expansions [12]. To further improve the performance for nonlinear problems, the UKF was proposed around 15 years ago [10]. It took quite a different route for propagation of covariance, as the traditional approach of Taylor expansions and focus on probability density functions (pdf's) was largely avoided. The method is based on the intuition that [11]: "it is easier to approximate a probability distribution than it is to approximate an arbitrary nonlinear function or transformation." The variant which will shortly be referred to as the UKF is readily described. The covariance of any model $h(q)$ which depends on uncertain parameters $q=\left(\begin{array}{llll}q_{1} & q_{2} & \cdots & q_{n}\end{array}\right)^{T}$, with mean $\langle q\rangle$ and $\operatorname{covariance} \operatorname{cov}(q)$, can be estimated as follows. Sample the statistical information in $2 n$ so-called sigma points $\sigma_{k}^{(s)}$ by calculating a square root of $\operatorname{cov}(q)$. That renders a deterministic ensemble,

$$
\begin{aligned}
\sigma_{k}^{(s)} & =\langle q\rangle+s \cdot \delta q^{(k)},\left\{\begin{array}{r}
k=1,2, \ldots n, \\
s= \pm,
\end{array}\right. \\
\delta q^{(k)} & =\sqrt{n} \cdot \Delta_{:, k}, \Delta \Delta^{T}=\operatorname{cov}(q) .
\end{aligned}
$$

The quantity $\Delta_{i, k}$ denotes the $k$ th column of $\Delta$. As for any finite ensemble, the mean $\langle h\rangle$ and the second moment around the mean $h_{\sigma}$, or any other statistical moment of the model $h(q)$ are then estimated with expectations $\langle\cdot\rangle_{\sigma}$ over all $2 n$ sigma points. In contrast to the EKF, the UKF estimates the scent, $\zeta=\langle h(\sigma)\rangle_{\sigma}-h\left(\langle\sigma\rangle_{\sigma}\right) \neq 0$. The covariance is correctly propagated for linear models. For nonlinear equations, the covariance is not correctly propagated, but usually considerably better than in the EKF, or LIN. A serious deficiency is that for many parameters with large covariance, the scaling with $\sqrt{n}$ may cause failure: The scaling is not related to the variability of the parameters, only their total number. The model may not be applicable for parametric variations beyond their range and may be strongly inaccurate, or even incorrect. The samples may be statistically (range of pdf) and/or physically (range of model) allowed. A possible solution is provided by the scaled unscented transformation [16]. There is no scaling problem for the UN approach to be proposed. It utilizes the minimum number of only two samples, i.e., $n=1$.

The sigma points of the UKF are however not unique. A candidate for $\Delta$ can be found by a Cholesky factorization [17]. Any linear transformation of its rows, $\Delta^{\prime}=\Delta U$ with only a 'half'-unitary matrix $U: U U^{T}=I$ ( $I$ being the identity matrix, $U^{T} U=I$ is not required), results in another equally valid matrix $\Delta^{\prime}$ but different sigma points, since $\Delta^{\prime} \Delta^{\prime T}=\Delta U[\Delta U]^{T}=\Delta U U^{T} \Delta^{T}=\Delta \Delta^{T}$. These half-unitary transformations can be used to also increase the size of the ensemble, which for other sampling rules sometimes might be required to obtain allowed samples [18]. The invariance to complete unitary transformations $\left(U: U^{T} U=U U^{T}=I\right)$ or arbitrary rotations will here be utilized to sample at confidence boundaries (Section 4).

\section{CONFIDENCE BOUNDARIES}

The confidence interval of the modeling result is here assumed to be of primary interest, rather than the second moment around the mean as in the UKF. For simplicity, the confidence interval of the model is here defined symmetrically with equal total probability above and below the interval. Asymmetric intervals require a trivial generalization which will be stated in Section 4.1.

\subsection{One Parameter}

The basic idea of our approach is most easily illustrated for a model $h$ depending on only one uncertain parameter. In Fig. 1, the assumed known pdf of the uncertain variable (top) and the propagated pdf of the model (bottom) are indicated.

The possible values of the parameter $q$ are indicated by the domain $Q$ in Fig. 1 . The parameters $q_{c}, h_{c}$ denote the centers of the probabilistically symmetric confidence intervals, neither the mean $\langle q\rangle,\langle h\rangle$ nor the corresponding medians. The half-widths of the intervals for confidence $p$ are labeled $q_{p}, h_{p}$, while $q_{\sigma}, h_{\sigma}$ (omitted in Fig. 1 for clarity) refer to standard deviations. They are related by coverage factors $k_{p}, q_{p}=k_{p}^{(q)} q_{\sigma}, h_{p}=k_{p}^{(h)} h_{\sigma}$. These factors differ for models nonlinear-in-parameters, $k_{p}^{(q)} \neq k_{p}^{(h)}$. The variations $\sigma^{( \pm)}=\langle q\rangle \pm q_{\sigma}$ are for obvious reasons called sigma points in the UKF. Here we are interested in confidence intervals and instead focus on the confidence boundaries $\eta^{( \pm)}: h\left(\eta^{( \pm)}\right)=h_{c} \pm h_{p}$. In Fig. 1, the upper and lower superscripts of $\eta$ relate to increasing ("I") and 


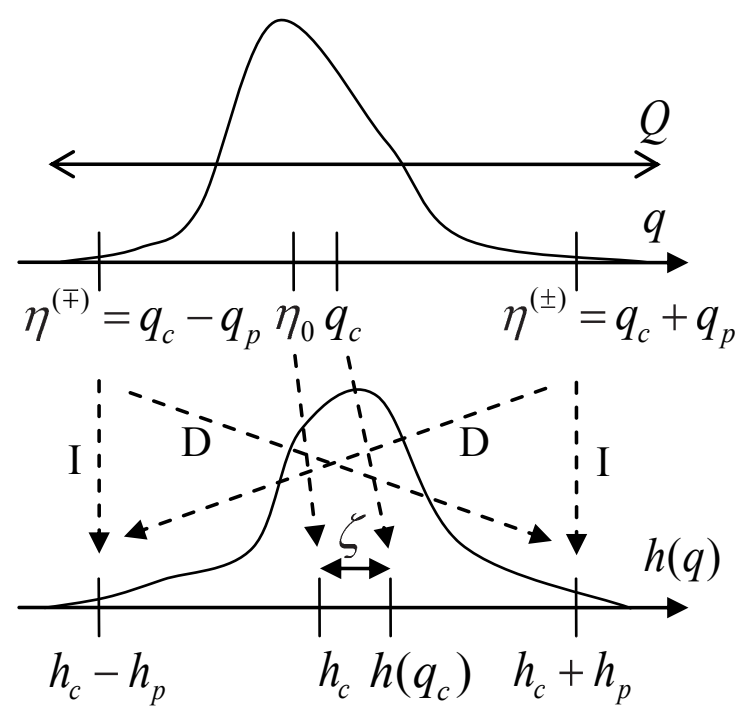

FIG. 1: The pdf of one uncertain parameter $q$ (top) propagated to the model $h$ (bottom). The notation is explained in the text.

decreasing ("D") $h$, respectively. The inverse centroid boundary $\eta^{(0)}: h\left(\eta^{(0)}\right)=h_{c}$ is also of interest since $\eta^{(0)}-q_{c}$ reflects the scent $\zeta=h_{c}-h\left(q_{c}\right)$. Ideally, the model $h$ is sampled at the lambda points $\lambda^{( \pm)} \in \eta^{( \pm)}$to directly give the exact confidence interval as $\left[h_{c}-h_{p}, h_{c}+h_{p}\right]=\left[h\left(\lambda^{(-)}\right), h\left(\lambda^{(+)}\right)\right]$.

If a model $h$ of one parameter $q$ is monotonic, it preserves or reverses the order: If $q_{1} \leq q_{2}, h\left(q_{1}\right) \leq h\left(q_{2}\right)$ or $h\left(q_{1}\right) \geq h\left(q_{2}\right), \forall q_{1}, q_{2} \in Q$. The symmetric $p$ percentiles of the variable $q$ are then mapped exactly to the $p$ percentiles of the mapping, either $h\left(q_{c} \pm q_{p}\right)=h_{c} \pm h_{p}$, or $h\left(q_{c} \pm q_{p}\right)=h_{c} \mp h_{p}$. The confidence boundaries can then be determined exactly, $\eta^{( \pm)}=q_{c} \pm q_{p}$ for increasing, and $\eta^{( \pm)}=q_{c} \mp q_{p}$ for decreasing $h$. Sampling at $\lambda^{( \pm)}=\eta^{( \pm)}$ then results in the exact confidence interval.

\subsection{Several Parameters and Uniqueness}

For two parameters, the confidence boundaries $\eta^{( \pm)}$are two lines. Hypothetical confidence boundaries are shown in Fig. 2. The associated confidence domain is defined by $\left\{q \in \Omega: \eta^{(-)}<h(q)<\eta^{(+)}\right\}$. Similar illustrations are included in the static examples in Sections 6.2 (Fig. 3) and 6.3 (Fig. 4).

The generalization to $n$ parameters is straightforward. The two confidence boundaries $\eta^{( \pm)}$will have dimension $n-1$ and the confidence domain $\Omega$ dimension $n$. Other types of confidence domains can be defined without any reference to a model. In that case they may not be uniquely defined and can have any topology, hyper-cubes or -ellipses, connected or not. The only requirement of such a confidence domain is to contain the correct accumulated probability. By defining the confidence boundaries of the model $h$ as $\eta^{( \pm)}: h\left(\eta^{( \pm)}\right)=h_{c} \pm h_{p}$, the confidence domain becomes uniquely defined and thus an object that can be estimated.

In one dimension, monotonicity of the model was central for finding the exact confidence boundaries. The geometry is more complex in higher dimensions and stronger restrictions on the model are required to solve the problem exactly. If the gradient exists almost everywhere and its direction is constant, the exact confidence boundaries can be determined for any number of parameters. This case proposes plausible approximations which will be utilized.

\section{SAMPLING ON CONFIDENCE BOUNDARIES}

Sampling on estimated confidence boundaries at $\lambda^{( \pm)} \in \hat{\eta}^{( \pm)} \approx \eta^{( \pm)}$will directly estimate the desired confidence interval of the model,

$$
\left[h_{c}-h_{p}, h_{c}+h_{p}\right] \equiv\left[h\left(\eta^{(-)}\right), h\left(\eta^{(+)}\right)\right] \approx\left[h\left(\lambda^{(-)}\right), h\left(\lambda^{(+)}\right)\right] .
$$




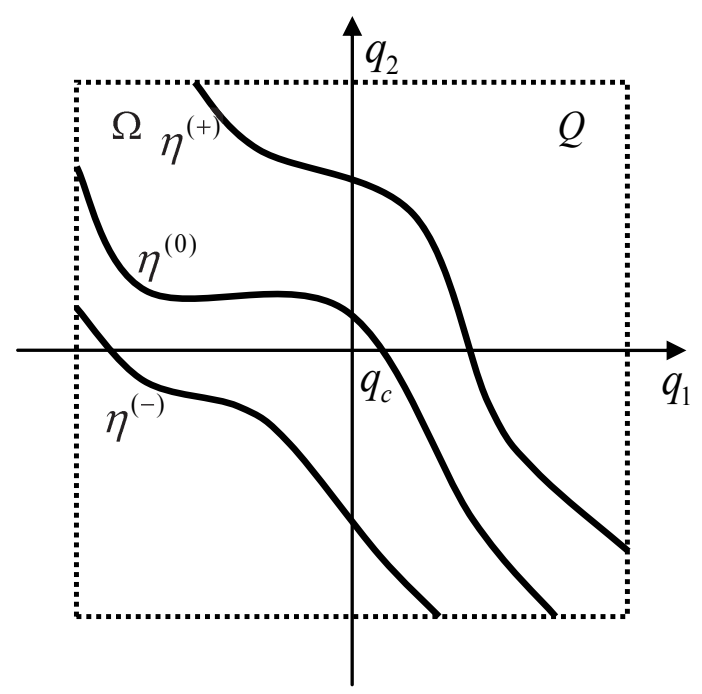

FIG. 2: Hypothetical confidence boundaries $\eta^{( \pm)}$and inverse centroid $\eta^{(0)}$ for two uncertain parameters. The notation is explained in the text.

Since it is generally not possible to determine $\eta^{( \pm)}$exactly, one has to resort to approximate methods such as the UNG and UNR methods to be described below. The statistical problem of determining the confidence interval of the model $h$ is effectively transformed to a geometric problem. The topology of the confidence domain is largely determined by the model structure, while the statistical variation of the parameters mainly affects its size. It is therefore appropriate to carefully study the complexity of the model structure, instead of focusing on the parametric uncertainty. That idea is fundamentally different to the approximating LIN and RSM, as well as the sampling UKF and LHS methods.

\subsection{The UNG Method}

As all points in each of the boundaries $\eta^{( \pm)}$are mapped to exactly the same value, the model is completely degenerate in all $n-1$ directions within each boundary. In the directions orthogonal to $\eta^{( \pm)}$, the variation of $h$ is maximal. All the $n-1$ degrees-of-freedom of a model in $n$ dimensions which span $\eta^{( \pm)}$may hence safely be ignored. The degeneracy is then removed from the model, which is thereby locally reduced to one dimension. The direction of maximal variation is however varying. One plausible approximation among others, is to neglect this variation and set the direction $\tilde{d}$ of maximum variation equal to the normalized gradient $\tilde{d} \equiv \tilde{\nabla}_{q} h=\nabla_{q} h /\left\|\nabla_{q} h\right\|$, evaluated at the center $q_{c}$. The boundaries $\eta^{( \pm)}$as well as $\eta^{(0)}$ are then approximated to be parallel. Still, $\eta^{(0)}$ may be anywhere between $\eta^{( \pm)}$and is able to describe a finite scent $\zeta$. The model will depend on only one nondegenerate "gradient parameter" $q_{\nabla} \equiv\left(\tilde{\nabla}_{q} h\right)^{T}\left(q-q_{c}\right)$, without any restriction on how it varies beyond monotonicity. Applying the standard UKF described in Section 2.3 results in two lambda points $\lambda^{( \pm)}$, corresponding to the sigma points $\sigma^{( \pm)}$. Expanded to the desired confidence level $p$, as suggested in Section 3.1,

$$
\begin{aligned}
\lambda^{(s)} & =q_{c}+s \cdot k_{p}^{(s)} \sigma \tilde{d}, \tilde{d}=\tilde{\nabla}_{q} h, \quad s= \pm, \\
\sigma & \equiv \sqrt{\tilde{d}^{T} \operatorname{cov}(q) \tilde{d}}
\end{aligned}
$$

where $k_{p}^{(s)}$ is the estimated coverage factor for the marginal probability distribution of $q_{\nabla}$. This is not a linear approximation of the model $h$ itself. Linearization is only made to estimate the topology but not the size of the confidence domain, and definitely not the magnitude of the model variation. A finite scent will indeed be obtained, contrary to the LIN method. The trivial generalization to account for asymmetric confidence intervals has here been made by allowing for different coverage factors in the two directions: For increasing models, $k_{p}^{(+)}$corresponds to the upper limit 
of the confidence interval for $q_{\nabla}$, and the reverse for $k_{p}^{(-)}$. For decreasing models, $k_{p}^{( \pm)}$should be interchanged. The confidence interval is finally obtained from Eq. (4). This constitutes our simplest method of Unscented Nondegenerate sampling with central Gradient approximation (UNG).

The scalar displacement $\sigma$ in Eq. (6) is the standard deviation of the marginal distribution of the only relevant (within the current approximation) parameter $q_{\nabla}$. It fuses the potential variation of the model $h$ with the actual variation of the parameters $q$. The propagation of covariance from $q$ to $q_{\nabla}=\left(\tilde{\nabla}_{q} h\right)^{T}\left(q-q_{c}\right)$ is exact since this transformation is linear. For the very same reason it is also simpler to estimate the coverage factor here than in the LIN method for which the coverage factor refers to the nonlinear model $h$. This is further explored in Section 4.4. Nonlinearities as well as the statistical information are thus generally more accurately described in the UNG, than in the LIN method.

\subsection{Higher Order Methods-The UNR Method}

More refined approximations than a constant gradient can be utilized to further increase the accuracy. Typically, sampling of the model is made twice in all UN methods:

1. Determine mu points $\left\{\mu^{(i)}, i=1,2, \ldots, j \geq n+1\right\}$ where the model will be tested for estimating confidence boundaries $\eta^{( \pm)}$. These will be the equivalent collocation points $[7,8]$.

2. Sample the model at collocation points $\left\{\mu^{(i)}\right\}$ to obtain $\left\{h\left(\mu^{(i)}\right)\right\}$.

3. Determine sampling points $\lambda^{(s)}$ from $\left\{h\left(\mu^{(i)}\right)\right\}, \operatorname{cov}(q)$, and estimated $k_{p}^{(s)}$.

4. Sample the model at $\lambda^{(s)}$ to obtain the estimated confidence limits $h\left(\lambda^{(s)}\right)$; see Eq. (4).

A simple difference quotient approximation [13] of the gradient is one example of this type of doubled sampling. The steps 1-2 are similar to but different from the first step of model approximation in RSM [9]. One difference is that the sampling of $\{\mu\}$ here is made to find local approximations of $h(q)$ at the boundaries $\eta^{( \pm)}$, not for the whole range of parameter values $q \in Q$. Instead of applying massive random sampling of the whole model approximation as in RSM, each confidence boundary is estimated in just one point in step 3. This allows for an absolutely minimal set of samples $\lambda^{(s)}$ but also an exact evaluation of the model $h$ in step 4 . The number $j$ can be chosen as large as is affordable by the model complexity and computational power. In total, $j+2 \geq n+3$ model evaluations are required for $n$ parameters.

An obvious generalization of the UNG method along these lines is to allow for nonparallel and more relevant estimates $\hat{\eta}^{( \pm)}$of confidence boundaries $\eta^{( \pm)}$. The lambda points $\lambda^{(s)}$ are then constructed with different directions $\tilde{d}^{( \pm)}$of maximum variation from $q_{c}$ to $\hat{\eta}^{( \pm)}$: The ascending direction $\tilde{d}^{(+)}$indicates the direction of largest increase of the model $h$ and is used to assign $\lambda^{(+)}$, while the descending direction $\tilde{d}^{(-)}$of largest decrease gives $\lambda^{(-)}$. These directions can be found with linear approximations on two different subsets of samples at $M^{(s)}=\left\{\mu^{(s, 0)}=\right.$ $\left.q_{c}, \mu^{(s, 1)}, \mu^{(s, 2)}, \ldots, \mu^{(s, k)}, k \geq n\right\}$, where $q_{c}$ is included as the reference point. Any sufficient number of $\mu$ samples may be used to find good robust estimates of $\tilde{d}^{(s)}$. Linear regression is here the obvious generalization of the difference quotient approximation of a gradient [13], to determine the offsets $h_{0}^{(s)}$ and directions $\tilde{d}^{(s)}$ [Eq. (7)],

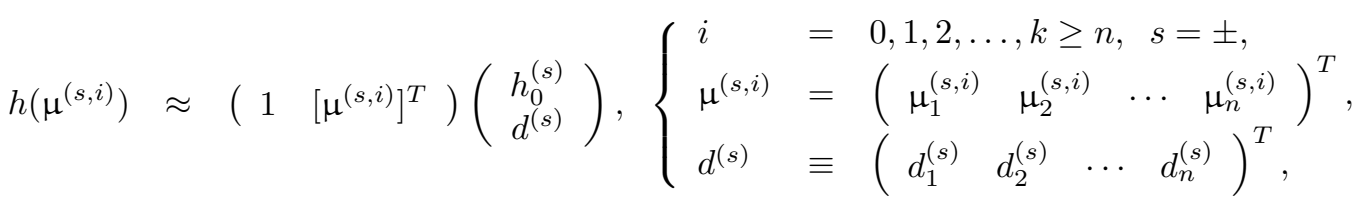

$$
\begin{aligned}
& \lambda^{(s)}=q_{c}+k_{p}^{(s)} \sigma^{(s)} \tilde{d}^{(s)}, \quad \tilde{d}^{(s)} \equiv d^{(s)} /\left\|d^{(s)}\right\|, \\
& \sigma^{(s)} \equiv \sqrt{\left[\tilde{d}^{(s)}\right]^{T} \operatorname{cov}(q) \tilde{d}^{(s)}} .
\end{aligned}
$$

Finally, specific rules for selecting $\left\{\mu^{(i)}\right\}$ and subsets $M^{(s)}$ are needed. To account for correlations and be as relevant as possible, select $\left\{\mu^{(i)}\right\}$ along the principal directions of $\operatorname{cov}(q)$ and as close as possible to potential confidence 
boundaries (without knowledge of $h$ ). That is, diagonalize $\operatorname{cov}(q)$ by finding a unitary matrix $U: U^{T} U=U U^{T}=I$ such that $U \operatorname{cov}(q) U^{T}$ is diagonal and let $\left(\mu^{(i)}\right.$ be given by column $i$ of $\left.\{\mu\}\right)$,

$$
\{\mu\}=q_{c}+\left\langle k_{p}^{(s)}\right\rangle_{s} U^{T} \sqrt{U \operatorname{cov}(q) U^{T}} V, V=\left(\begin{array}{ll}
I & -I
\end{array}\right) .
$$

The sets $M^{(s)}$ can be chosen by first sorting $\{\mu\}$ into $\left\{\mu^{(i)}: h\left(\mu^{(1)}\right) \leq h\left(\mu^{(2)}\right) \leq \cdots \leq h\left(\mu^{(2 n)}\right)\right\}$. Then, extract $M^{(-)}=\left\{q_{c}, \mu^{(1)}, \mu^{(2)}, \ldots, \mu^{(n+m)}\right\}$ and $M^{(+)}=\left\{q_{c}, \mu^{(n+1-m)}, \mu^{(n+2-m)}, \ldots, \mu^{(2 n)}\right\}$. If $m=0$ is chosen, minimal sets $M^{(s)}$ are used and Eq. (7) yields a perfect linear approximation. If the regression matrix with row $i$ given by $\left(1\left[\mu^{(s, i)}\right]^{T}\right)$ happens to be rank deficient, the regression becomes ill-conditioned. To obtain a definite direction $d^{(s)}$, either use the Moore-Penrose pseudoinverse [17] of the regression matrix, or let $m>0$. If $m=n$, $M^{(-)}=M^{(+)}$, giving $d^{(-)}=d^{(+)}=d$. The UNR method is then reduced to the UNG method with a secant approximation of the gradient, $\nabla_{q} h \approx d$. This completes the method of Unscented Nondegenerate sampling using linear Regression (UNR). It is applicable for any number of parameters, for static as well as dynamic models, and is illustrated in Sections 6.3 and 6.4.

\subsection{Accuracy of UNG and UNR}

The UNG and UNR approximations belong to a class of methods based on Unscented Nondegenerate sampling (UN) at confidence boundaries which has no generic error associated to it. Common to all UN methods is that all errors are due to incorrect determination of the lambda points. The discussion of accuracy here will therefore be limited to errors $\delta \lambda^{(s)}$ of $\lambda^{(s)}$. The model $h$ will be assumed to be at least twice differentiable.

\subsubsection{Constant Gradient Direction}

Both UNG and UNR methods approximate the gradient $\nabla h$ to have a constant direction $\tilde{d}$ when calculating $\lambda$. Their accuracy can be estimated by first assuming a constant gradient direction $\tilde{\nabla} h$, but a limited directional error $\delta \tilde{d}=$ $\tilde{d}-\tilde{\nabla} h,|\delta \tilde{d}| \ll 1$, in the expressions for $\lambda$. The appropriate gradient $\tilde{\nabla} h$ to use below will be derived in Section 4.3 .2 . To lowest order,

$$
\frac{\delta \lambda^{(s)}}{\left|\lambda^{(s)}-q_{c}\right|}=\frac{\delta\left(q_{c}+k_{p} \sigma \tilde{d}^{(s)}\right)}{k_{p} \sigma}=\frac{\delta \sigma}{\sigma} \tilde{d}^{(s)}+\delta \tilde{d}^{(s)}
$$

Even though the only error is due to incorrect direction $\tilde{d}^{(s)}, \lambda^{(s)}$ is defective in both magnitude (first term) and direction (second term), relative to $q_{c}$. Since variations within the confidence boundaries do not influence the model, project $\delta \lambda^{(s)}$ on $s \tilde{\nabla} h$ to find the relevant error (sign included) of $\lambda^{(s)}$. Being a relative error it also valid for $\delta \lambda^{(s)}$ calculated along $\tilde{d}^{(s)}$,

$$
\frac{\delta \lambda_{\|}^{(s)}}{\left|\lambda^{(s)}-q_{c}\right|}=\left[\frac{\delta \sigma}{\sigma}[s \tilde{\nabla} h]^{T} \tilde{d}^{(s)}+[s \tilde{\nabla} h]^{T} \delta \tilde{d}^{(s)}\right] \tilde{d}^{(s)}=s\left[\frac{\sqrt{\left[\tilde{d}^{(s)}\right]^{T} \operatorname{cov}(q) \tilde{d}(s)}}{\sqrt{[\tilde{\nabla} h]^{T} \operatorname{cov}(q) \tilde{\nabla} h}}[\tilde{\nabla} h]^{T} \tilde{d}^{(s)}-1\right] \tilde{d}^{(s)}
$$

To further analyze the error, make an eigenvalue decomposition, $\operatorname{cov}(q)=U^{T} \Sigma^{2} U, \Sigma_{j k}=\delta_{j k} \bar{\sigma}_{k}$, where $\bar{\sigma}_{k}$ is the standard deviation in uncorrelated parameters $U q$. Then,

$$
\frac{\delta \lambda_{\|}^{(s)}}{\left|\lambda^{(s)}-q_{c}\right|}=s\left[\frac{\left|\Sigma U \tilde{d}^{(s)}\right|}{|\Sigma U \tilde{\nabla} h|}[\tilde{\nabla} h]^{T} \tilde{d}^{(s)}-1\right] \tilde{d}^{(s)} .
$$

Since there is no preferred basis, the evaluation may equally well be made in a canonical basis $\bar{q}=\Sigma^{-1} U q$ for which $\operatorname{cov}(\bar{q})=I$. The canonical basis is not unique if $\operatorname{cov}(q)$ is degenerate, i.e., at least two of its eigenvalues 
are identical. Any such basis is appropriate here. The normalized gradient and direction (assuming it transforms as the gradient), evaluated in a canonical basis, but expressed in the original basis, read $\bar{\nabla} h=\Sigma U \tilde{\nabla} h /|\Sigma U \tilde{\nabla} h|$ and $\bar{d}^{(s)}=\Sigma U \tilde{d}^{(s)} /\left|\Sigma U \tilde{d}^{(s)}\right|$. Then, $|\Sigma U \tilde{\nabla} h|=\left|\Sigma U \tilde{d}^{(s)}\right|=1$ due to normalization of $\bar{\nabla} h$ and $\bar{d}^{(s)}$,

$$
\frac{\delta \bar{\lambda}_{\|}^{(s)}}{\left|\bar{\lambda}(s)-q_{c}\right|}=s\left[(\tilde{\nabla} h)^{T} \tilde{d}^{(s)}-1\right] \tilde{d}^{(s)}=R\left(|\delta \tilde{d}|^{2}\right) \tilde{d}^{(s)}
$$

where $R(x)$ is of order $x$. The first-order contribution to the error is thus eliminated if $\lambda^{(s)}$ is evaluated in a canonical basis. Evaluation in a canonical basis is consequently prescribed if ||$\Sigma U \tilde{d}^{(s)}|-| \Sigma U \tilde{\nabla} h \|>R\left(\left|\delta \tilde{d}^{(s)}\right|^{2}\right)$, i.e., $\left|\sqrt{\left[\tilde{d}^{(s)}\right]^{T} \operatorname{cov}(q) \tilde{d}^{(s)}}-\sqrt{[\tilde{\nabla} h]^{T} \operatorname{cov}(q) \tilde{\nabla} h}\right|>R\left(\left|\delta \tilde{d}^{(s)}\right|^{2}\right)$. This can only happen for $\left|\delta \tilde{d}^{(s)}\right| \ll 1$ if $\min _{k} \bar{\sigma}_{k} \ll$ $\max _{k} \bar{\sigma}_{k}$.

The lambda points may be evaluated in a canonical basis without formally changing the basis. The modified lambda points $\bar{\lambda}^{(s)}$ should then be expressed in the gradient $\tilde{\nabla} h$ and the direction $\tilde{d}^{(s)}$ in the original basis with parameters $q$,

$$
\bar{\lambda}^{(s)}=q_{c}+k_{p} U^{T} \Sigma\left(\left|\Sigma U \tilde{d}^{(s)}\right|^{-1} \Sigma U \tilde{d}^{(s)}\right)=q_{c}+\frac{k_{p}}{\sqrt{\left[\tilde{d}^{(s)}\right]^{T} \operatorname{cov}(q) \tilde{d}^{(s)}}} \operatorname{cov}(q) \tilde{d}^{(s)} .
$$

The original direction $\tilde{d}^{(s)}$ is rotated and rescaled - the magnitude of the lambda-point variation $\lambda^{(s)}-q_{c}$ changes to

$$
\left|\bar{\lambda}^{(s)}-q_{c}\right|=k_{p}\left|\Sigma U \tilde{d}^{(s)}\right|^{-1}\left|\Sigma^{2} U \tilde{d}^{(s)}\right| \neq k_{p}\left|\Sigma U \tilde{d}^{(s)}\right|=\left|\lambda^{(s)}-q_{c}\right| .
$$

If either $\left(U \tilde{d}^{(s)}\right)_{j k}=\delta_{j k}$ or $\Sigma \propto I$, the variations are equal. In both cases, the scaling with $\Sigma$ does not change the direction $U \tilde{d}^{(s)}$. To simplify the presentation, calculation in a canonical basis is not considered (or required) in any example in Section 6. For evaluation in a canonical basis, simply use Eq. (15) instead of Eqs. (5) and (8) for evaluating $\lambda^{(s)}$.

\subsubsection{Variable Gradient Direction}

When the gradient direction varies over $\Omega$, the lambda points will be defective for two reasons: (1) The different level hypersurfaces of the model are not parallel. (2) Each hypersurface is bent or buckled. In short, the gradient directions vary between (1) as well as within (2) the hypersurfaces. The directional variation between different hypersurfaces (1) makes it difficult to propagate the covariance exactly, as the traditional approach [1] prescribe. In the absence of buckling (2), the confidence boundaries may nevertheless be determined exactly. The reason is that each confidence boundary is only dependent on a single-level hypersurface $h(q \in \eta)=h(\lambda)$, while the covariance relates to all of them. The proper direction for calculating $\lambda^{(s)}$ is consequently $\tilde{d}^{(s)}=s \tilde{\nabla} h\left(\lambda^{(s)}\right)$. In detail, this can be understood as follows. With the transformation $q \rightarrow q_{d} \equiv\left[\tilde{d}^{(s)}\right]^{T}\left(q-q_{c}\right)$, the multi variate pdf $f(q)$ is marginalized to $f_{d}\left(q_{d}\right)$ over all degrees of freedom orthogonal to $\tilde{d}^{(s)}$ (temporarily omitting $s$ to simplify notation),

$$
f_{d}\left(q_{d} \geq 0\right) \equiv \int_{S_{\perp}\left(q_{d}\right)} f\left(q_{1}, q_{2}, \ldots, q_{n}\right) d \hat{q}_{1} d \hat{q}_{2} \ldots d \hat{q}_{n-1}, \quad\left\{\hat{q} \in S_{\perp}\left(q_{d}\right): d^{T}\left(\hat{q}-q_{c}\right)=q_{d}\right\} .
$$

The univariate pdf $f_{d}\left(q_{d}\right)$ also has an associated coverage factor $k_{p}(\tilde{d})$. Considering both directions $\tilde{d}^{(s)}$, the region $\left\{q \in \Omega^{(+)}:\left[d^{(s)}\right]^{T} q \geq 0, s= \pm\right\}$ will be integrated twice while the domain $\left\{q \in \Omega^{(-)}:\left[d^{(s)}\right]^{T} q \leq 0, s= \pm\right\}$ is omitted. However, if $k_{p}\left(\tilde{d}^{(s)}\right)=k_{p}$, there will be no error: By mirror imaging through $q_{c}, q-q_{c} \rightarrow-\left(q-q_{c}\right)$, the integration over $\Omega^{(+)}$will be equivalent to an integration over $\Omega^{(-)}$. The covariance matrix is left invariant by this transformation as it has a quadratic dependence on $q-q_{c}$. If also $k_{p}\left(\tilde{d}^{(s)}\right)$ is independent of $\tilde{d}^{(s)}$, the integrations over 
the domains $\Omega^{(s)}$ are exchangeable so the errors cancel exactly. Thus for each region defined by $q_{d} \geq 0$, integrate the marginalized pdf $f_{d}$ to the boundary $q_{d}=\left[\tilde{d}^{(s)}\right]^{T}\left(\lambda-q_{c}\right)=\eta$ to yield the probability $P / 2$,

$$
\frac{P}{2}=\int_{0}^{\eta} f_{d}\left(q_{d}\right) d q_{d}
$$

If $\tilde{d}^{(s)}=\tilde{\nabla} h\left(\left\{q: q_{d}=\eta^{(s)}\right\}\right)=\tilde{\nabla} h\left(\lambda^{(s)}\right), h\left(q_{1}: q_{d}=\eta^{(s)}\right)=h\left(q_{2}: q_{d}=\eta^{(s)}\right), \forall q_{1}, q_{2} \in Q^{(s)}$. Hence, the boundary $q_{d}=\eta^{(s)}$ is a level hypersurface of the model $h$, i.e., the correct confidence boundary. In other words: (1) The hypersurface for $h\left(\lambda^{(s)}\right)$ must be planar. (2) The direction $\tilde{d}^{(s)}$ coincides with $s \tilde{\nabla} h\left(\lambda^{(s)}\right)$ at the estimated confidence boundary. Clearly, UNR may be much more accurate than UNG, as it is possible to have $d^{(-)} \neq d^{(+)}$ in UNR but not in UNG. The magnitude error due to incorrect directions $\tilde{d}^{(s)}$ for calculating $\lambda^{(s)}$ can be determined from Eq. (12) by evaluating the gradient at the lambda points, $\tilde{\nabla} h=\tilde{\nabla} h\left(\lambda^{(s)}\right)$. Note that with varying direction of the gradient, an incorrect direction $\tilde{d}^{(s)}$ also will result in an incorrect estimate of the gradient at the confidence boundary, $\tilde{\nabla} h\left(\lambda^{(s)}\right) \neq \tilde{\nabla} h\left(\eta^{(s)}\right)$. If the direction of the gradient has a moderate variation over $\delta \tilde{d}$, the error will be limited, as illustrated in examples in Sections 6.2 and 6.3.

\subsubsection{Buckled Confidence Boundaries}

If $\lambda^{(s)}$ is determined for the correct direction $\tilde{d}^{(s)}=s \tilde{\nabla} h\left(\lambda^{(s)}\right)$, all errors are due to buckling of the level hypersurfaces given by $h\left(q \in Q^{(s)}\right)=h\left(\eta^{(s)}\right) \approx h\left(\lambda^{(s)}\right)$. Buckling of a level surface is here defined by the variation of the gradient direction within the level surface. An upper bound of the error $\delta \lambda_{B}^{(s)}$ of the lambda point $\lambda^{(s)}$ due to this buckling is readily estimated. First, expand the model variation $\delta h$ around $q=\lambda^{(s)}$ to second order,

$$
\delta h^{(s)}=\left[\nabla h\left(\lambda^{(s)}\right)\right]^{T} \delta q+\frac{1}{2}[\delta q]^{T} H\left(\lambda^{(s)}\right) \delta q+R\left(|\delta q|^{3}\right), \delta q=q-\lambda^{(s)},
$$

where $H\left(\lambda^{(s)}\right)$ is the Hessian matrix of $h$, evaluated at $\lambda^{(s)}$. Define the plane $\eta_{\perp}^{(s)}$ to be tangent to the buckled surface $\eta^{(s)}$ at $q=\lambda^{(s)}$. Now maximize the transverse variation $\left|\delta h_{\perp}^{(s)}\right|$ by varying $\delta q_{\perp}^{(s)} \equiv q-\lambda^{(s)} \in \eta_{\perp}^{(s)}$ over the whole allowed range $q \in \eta_{\perp}^{(s)} \cap Q$. To estimate the buckling of the level surface $h\left(\lambda^{(s)}\right)$, also find a compensating longitudinal model variation $\delta h_{\|}^{(s)}=-\delta h_{\perp}^{(s)}$ with the variation $\delta q_{\|}^{(s)} \equiv q-\lambda^{(s)}:\left[\delta q_{\|}^{(s)}\right]^{T} \delta q_{\perp}^{(s)}=0$. Switching from the approximate $\left(\eta_{\perp}^{(s)}\right)$ to the true boundary $\left(\eta^{(s)}\right)$ while maintaining the enclosed probability between the corresponding surfaces for $s= \pm, \lambda^{(s)}$ has to be adjusted by an amount $\left|\delta \lambda^{(s)}\right|<\left|\delta q_{\|}^{(s)}\right|$. By assuming a (locally) uniform probability distribution, using a quadratic model of buckling as in Eq. (19), and equalizing the two integrations of probability for the planar and buckled surfaces, the bound can be narrowed down to $\left|\delta \lambda^{(s)}\right| \leq\left|\delta q_{\|}^{(s)}\right| / 3$. This will yield an upper bound of the magnitude of the lambda-point error $\left|\delta \lambda^{(s)}\right|$ due to buckling. To also account for the sign of $\delta \lambda^{(s)}$, use $\delta \lambda_{B}^{(s)} \equiv \delta\left|q_{\|}^{(s)}\right| / 3, \delta\left|q_{\|}^{(s)}\right| \equiv \operatorname{sign}\left(\delta q_{\|}^{(s)}\right)\left|\delta q_{\|}^{(s)}\right|$ as a conservative measure of the buckling error of $\lambda^{(s)}$. While this provides the principle for how the error can be estimated, the relevant quantities will be evaluated below.

For the transverse variation, $[\nabla h]^{T} \delta q_{\perp}^{(s)}=0$ in Eq. (19). A rough estimate of the relevant range of $\delta q_{\perp}^{(s)}$ in direction $\tilde{d}_{\perp}^{(s)} \in \eta_{\perp}^{(s)}$ is given by the (marginalized) standard deviation $\sigma_{\perp}^{(s)}=\sqrt{\left[d_{\perp}^{(s)}\right]^{T} \operatorname{cov}(q) d_{\perp}^{(s)}}$. To find the largest relevant buckling, the corresponding model variation in Eq. (19) should be maximized for $\delta q_{\perp}^{(s)}=\sigma_{\perp}^{(s)} \tilde{d}_{\perp}^{(s)}$,

$$
\max \left|\delta h_{\perp}^{(s)}\right|=\frac{1}{2} \max _{\left|\tilde{d}_{\perp}^{(s)}\right|=1}\left|\left[\tilde{d}_{\perp}^{(s)}\right]^{T} \operatorname{cov}(q) \tilde{d}_{\perp}^{(s)}\left[\tilde{d}_{\perp}^{(s)}\right]^{T} H \tilde{d}_{\perp}^{(s)}\right|, \tilde{d}_{\perp}^{(s)} \in \eta_{\perp}^{(s)} \cap Q .
$$

This constrained quartic (4) form of $\tilde{d}_{\perp}^{(s)}$ may be converted to a simpler quadratic (2) form by changing to canonical parameters satisfying $\operatorname{cov}(\bar{q})=1$, as in Section 4.3.2. The Hessian transforms according to how the gradient transforms, $\left.H=\left[\nabla \otimes \nabla^{T}\right] h \rightarrow \bar{H}=\left[(\Sigma U \nabla) \otimes(\Sigma U \nabla)^{T}\right)\right] h$, where $\otimes$ denotes outer product,

$$
\max \left|\delta h_{\perp}^{(s)}\right|=\frac{1}{2} \max _{\left|\bar{d}_{\perp}^{(s)}\right|=1}\left|\left[\bar{d}_{\perp}^{(s)}\right]^{T} \bar{H} \bar{d}_{\perp}^{(s)}\right|, \bar{d}_{\perp}^{(s)} \in \eta_{\perp}^{(s)} \cap Q, \bar{H} \equiv \Sigma U H U^{T} \Sigma .
$$


To satisfy $\bar{d}_{\perp}^{(s)} \in \eta_{\perp}^{(s)}$, express the variation in an orthonormal (ON) basis contained in $\eta_{\perp}^{(s)}$. It can be obtained by projecting the unit basis vectors on $\eta_{\perp}^{(s)}$, or equivalently, by subtracting their projections on the gradient. This will result in $n$ vectors in $\eta_{\perp}^{(s)}$. These are neither mutually orhogonal nor normalized but span $\eta_{\perp}^{(s)}$ with $n-1$ degrees of freedom. One vector becomes redundant after the projection. To eliminate this redundancy and convert the remaining vectors to an ON-basis $E^{(s)}$ with basis vectors in columns $e_{k}, k=1, \ldots, n-1$, apply Gram-Schmidt orthogonalization [14]. It will here be formulated in terms of an operator $G$, which includes elimination of redundancy. It is easily identified, as redundant vectors will vanish in the orthogonalization. In the canonical basis used here,

$$
E^{(s)}=G\left(I-\bar{\nabla} h^{(s)} \otimes\left[\bar{\nabla} h^{(s)}\right]^{T}\right)=G\left(I-\left|\Sigma U \tilde{\nabla} h^{(s)}\right|^{-2} \Sigma U \tilde{\nabla} h^{(s)} \otimes\left[\tilde{\nabla} h^{(s)}\right]^{T} U^{T} \Sigma\right) .
$$

Expressed in this basis, $\bar{d}_{\perp}^{(s)}=E \hat{d}_{\perp}^{(s)}$, where $\hat{d}_{\perp}^{(s)}$ is the column vector of coordinates in the $E$ basis. If the transformed Hessian is also diagonalized, Eq. (21) reads

$$
\begin{aligned}
\max \left|\delta h_{\perp}^{(s)}\right| & =\frac{1}{2} \max _{\left|\hat{d}_{\perp}^{(s)}\right|=1}\left|\left[\hat{d}_{\perp}^{(s)}\right]^{T} \hat{H} \hat{d}_{\perp}^{(s)}\right|, \hat{H} \equiv E^{T} \Sigma U H U^{T} \Sigma E=\hat{V}^{T} \hat{\Gamma} \hat{V} \\
\hat{\Gamma}_{j k} & =\delta_{j k} \hat{\gamma}_{k}, \hat{V}^{T} \hat{V}=\hat{V} \hat{V}^{T}=I .
\end{aligned}
$$

The constraint $\bar{d}_{\perp}^{(s)} \in \eta_{\perp}^{(s)}$ has here been eliminated by one additional transformation $E$. According to the spectral theorem [14], diagonalization of the symmetric matrix $\hat{H}$ is always possible and the eigenvalues $\hat{\gamma}_{k}$ are real-valued. Since also $\left|\hat{d}_{\perp}^{(s)}\right|=1$, the largest variation is given by the eigenvalue with the largest magnitude,

$$
\max \left|\delta h_{\perp}^{(s)}\right|=\frac{1}{2} \max _{k}\left|\hat{\gamma}_{k}\right|, k=1,2, \ldots, n-1 .
$$

For the longitudinal variation, $[\nabla h]^{T} \delta q_{\|}^{(s)} \neq 0$ in Eq. (19). A linear model approximation is then presumably sufficient. The variation is here along the gradient direction, $\delta q_{\|}^{(s)}=\delta\left|q_{\|}^{(s)}\right| s \tilde{\nabla} h\left(\lambda^{(s)}\right)$, giving the model variation

$$
\delta h_{\|}^{(s)}=\left[\nabla h\left(\lambda^{(s)}\right)\right]^{T} \delta q_{\|}^{(s)}=s|\nabla h| \delta\left|q_{\|}^{(s)}\right| .
$$

Inferring $\delta h_{\perp}^{(s)}=-\delta h_{\|}^{(s)}$ and $\delta \lambda_{B} \equiv \delta\left|q_{\|}^{(s)}\right| / 3$ as argued above, upper bounds for the lambda-point errors due to buckling of the confidence boundaries are found,

$$
\frac{\delta \lambda_{B}^{(s)}}{\left|\lambda^{(s)}-q_{c}\right|}=-\frac{s}{6} \frac{\max _{k}\left|\hat{\gamma}_{k}\right|}{|\nabla h| k_{p} \sqrt{[\tilde{\nabla} h]^{T} \operatorname{cov}(q) \tilde{\nabla} h}} .
$$

\subsection{Coverage Factors}

All studied approximate methods (LIN, UNG, UNR) require estimates of coverage factors. For all UN methods, removing the degeneracy is equivalent to marginalization [19] of the multivariate distribution of $q-q_{c}$ into a univariate distribution over a directional parameter $q_{d}=\tilde{d}^{T}\left(q-q_{c}\right)$ (in simplified notation). Instead of estimating coverage factors $k_{p}$ and propagating covariance as in Eqs. (5)-(6) or (8)-(9), each sample point $\lambda=q_{c}+h_{d} \tilde{d}$ may be directly determined from the marginal distribution of $q_{d}$. The step length $h_{d}$ may easily be found numerically with rudimentary MC:

1. Diagonalize the covariance matrix, i.e., find $U: U^{T} U=U U^{T}=I$ such that $U \operatorname{cov}(q) U^{T}$ is diagonal.

2. Generate a random multivariate ensemble of $m$ samples of $n$ independent parameters and collect in matrix $W$ of size $n \times m,\left\{W: W W^{T} / m=U \operatorname{cov}(q) U^{T}\right\}$. 
3. Transform and marginalize $\left\{q-q_{c}\right\}=U^{T} W$ along direction $\tilde{d}$ to obtain the univariate ensemble (row vector) $D=\tilde{d}^{T} U^{T} W$.

4. Sort $D$ and extract percentile $h_{d}$.

Since no evaluation of the complex model is needed and the marginalization only consists of a linear transformation, random sampling is in this case numerically very inexpensive. The error of evaluating $k_{p}$ may thus in practice be reduced to a minimum for all UN methods, for static as well as dynamic models. That is important since the estimation of $k_{p}$ may be a dominating source of calculation errors.

In stark contrast, the coverage factor of the LIN method relates to the complex model. Thus, there is no equivalent effective numerical method to find $k_{p}$. It must instead be estimated, or rather guessed, from general aspects of the model parameters and their distribution [1]. In particular, for a dynamic measurement it must be assigned to a constant, despite it is varying in time; see Fig. 7. This will directly render a lower bound of the error of evaluating $h_{p}$, as argued in Section 6.4.2.

\section{DYNAMIC MODELS}

Evaluation of dynamic models [20] is synonymous to dynamic simulations over an entire time epoch: The models are ordinary differential equations in the time domain, which can be expressed in terms of transfer functions $G(q, s)$, or $G(q, z)$. These functions are the Laplace- or $z$ transforms of impulse responses $g(q, t)$. A dynamic model $h(y, q, t)$ is evaluated by convolving $(*)$, the impulse response of the system $g(q, t)$, with its input signal $y(t), h(y, q, t)=g(q, t) *$ $y(t)$. The model $h(y, q, t)$ is thus an implicit function of time $t$ via the whole signal $y(t)$ and not only its instantaneous amplitude $y(t)$ (as for static models). The model system $g(q, t)$ as well as the gradient system $\nabla_{q} g(q, t)$ can often be realized with digital filters [18]. Due to imposed correlations of the dynamic system $g(q, t)$, most quantities become generically time-dependent signals or objects, the $n$-dimensional gradient $\nabla_{q} h(y, q, t)=\left[\nabla_{q} g(q, \tau) * y(\tau)\right]_{\tau=t}$, the confidence boundaries $\eta^{( \pm)}(t)$, as well as the coverage factor $k_{p}(t)$. The lambda points $\lambda^{(s)}(t)$ in Eq. (4) are thus unique for each time instant $t$. The gradient column vector $\nabla_{q} h$ is for dynamic models conveniently generalized to a matrix of sensitivity signals arranged in rows, its element $\left[\nabla_{q} h\right]_{k l}=\left[\nabla_{q} h\left(y, q, t_{l}\right)\right]_{k}$ is the sensitivity for parameter $q_{k}$ at time $t_{l}$.

Throwing away all evaluated samples for every pair of lambda points except at $t$ makes UN sampling potentially inefficient but is an unavoidable consequence of the time dependence of $\eta^{( \pm)}(t)$. Fortunately, the response times $\tau\left(\lambda^{(s)}\right)$ of the impulse responses $g\left(\lambda^{(s)}(t), t\right)$ are for all practical purposes finite, also for infinite impulse response (IIR) systems. Therefore it is sufficient to apply the filters from $t-\tau\left(\lambda^{(s)}\right)$. For asymmetric time-reversed correction $[18,21]$, there are two response times, $\tau_{D}\left(\lambda^{(s)}\right)$ for direct and $\tau_{R}\left(\lambda^{(s)}\right)$ for time-reversed filtering. We are then allowed to only apply the digital filters $g\left(\lambda^{(s)}(t), t\right)$ to $y(t)$ for $t-\tau_{D}\left(\lambda^{(s)}\right) \leq t \leq t+\tau_{R}\left(\lambda^{(s)}\right)$. This is usually a minute fraction of the whole time interval $t$.

Applying the LIN method on dynamic measurements [18, 22], the center $h_{c}$ and half-width $h_{p}$ of the confidence interval at time $t_{l}$ are given by

$$
\begin{aligned}
& h_{c}\left(t_{l}\right)=\left[g\left(q_{c}, t\right) * y(t)\right]_{t=t_{l}}, \\
& h_{p}\left(t_{l}\right)=k_{p} \sqrt{\nabla_{q} h\left(y, q_{c}, t_{l}\right)^{T} \operatorname{cov}(q) \nabla_{q} h\left(y, q_{c}, t_{l}\right)} .
\end{aligned}
$$

As stated in Section $3.1, k_{p}^{(h)} \neq k_{p}^{(q)}$. Consistency requires the same model approximation (linearization) for estimating $k_{p}$ as for propagating $\operatorname{cov}(q)$. That erroneously implies $k_{p}^{(h)}=k_{p}^{(q)}$ for LIN. The induced temporal correlations in a dynamic measurement also cause $k_{p}^{(h)}(t)$ but not $k_{p}^{(q)}$ to be time-dependent. Both nonlinearities-in-parameters and dynamic effects are thus error sources of $k_{p}$ in LIN but neither of them results in errors of $k_{p}$ in the UN methods.

\section{EXAMPLES}

The examples are not selected to be representative for the complexity of typical applications, but rather to provide simple and transparent illustrations of the performance of UN methods. Throughout, the confidence level will be 
chosen equal to $95 \%$ with symmetric intervals (equal probability below and above the interval). For all static examples, the distributions of all parameters are assumed normal. The distribution of $q_{d}=\tilde{d}^{T} q$ is then also normal $\forall \tilde{d}$. That solves the problem of estimating coverage factors for all studied methods, $k_{p}=1.96$. This also allows for simple and straightforward comparisons to MC simulations, as a diagonalization of $\operatorname{cov}(q)$ also results in independent parameters. The samples may thus be correctly created by unitary transformations of random samples of independent parameters. In the dynamic example the parameters are bounded. Therefore, a uniform distribution is chosen. A bonus of this choice is that it will illustrate the time dependence of coverage factors in dynamic measurements, and that they can be estimated with the proposed UN methods. To further emphasize nonlinear effects and differences between various methods, the covariance matrices are set very large.

\subsection{Static Model 1}

An illustrative example of a simple static model equation is

$$
\begin{array}{lll}
h & =\left(q_{1}+q_{2}\right)^{3} \\
q_{c} & =(0,0)^{T} \\
\operatorname{cov}(q) & =\left(\begin{array}{cc}
0.104 & -0.019 \\
-0.019 & 0.196
\end{array}\right) .
\end{array}
$$

The LIN method is clearly a poor and also invalid approximation which results in zero uncertainty since the gradient $\nabla_{q} h=3\left(q_{1}+q_{2}\right)^{2}\left(\begin{array}{ll}1 & 1\end{array}\right)^{T}$ vanishes at $q_{c}$. For application of the UNG method, it is the normalized gradient that is of interest, $\tilde{\nabla}_{q} h=1 / \sqrt{2}\left(\begin{array}{ll}1 & 1\end{array}\right)^{T}, q_{1}+q_{2} \neq 0$. It does not exist along the line $q_{1}+q_{2}=0$ but may be continued to the same value it has in all other points. The direction of maximum variation is then constant. The lambda points will be

$$
\lambda^{( \pm)}= \pm k_{p}^{( \pm)} \sqrt{\left(\tilde{\nabla}_{q} h\right)^{T} \operatorname{cov}(q) \tilde{\nabla}_{q} h} \tilde{\nabla}_{q} h= \pm 0.501\left(\begin{array}{c}
1 \\
1
\end{array}\right) .
$$

According to Eq. (4), $\left[h_{c}-h_{p}, h_{c}+h_{p}\right]=\left[h\left(\lambda^{-}\right), h\left(\lambda^{+}\right)\right]=[-1.007,1.007]$. In this case the UNG method is exact: The direction of maximum variation is constant so there is no approximation to use a constant gradient to estimate the shape of the confidence domain.

This result may immediately be generalized: For any $h\left[w=a^{T}\left(q-q_{c}\right)\right],\left\{a, q_{c}, q\right\} \in \Re^{(n \times 1)}$, where $h(w)$ is monotonic and $q$ has a multivariate normal distribution, the UNG method is exact. The transformation to the gradient parameter $q_{\nabla}=\left(\tilde{\nabla}_{q} h\right)^{T}\left(q-q_{c}\right)=\|a\|^{-1} a^{T}\left(q-q_{c}\right)$ results in a strictly one-dimensional model $h\left(\|a\| q_{\nabla}\right)$. Disregarding the problem of determining the coverage factor, the UNG method is exact for all such one-dimensional (1D) models (see Section 3.1 and Fig. 1). In stark contrast, the LIN method is a more or less crude approximation whenever $h$ is nonlinear-in-parameters.

\subsection{Static Model 2}

Another closely related example of a static model is

$$
\begin{array}{ll}
h & =\alpha q_{1} q_{2}^{3}, \quad \alpha=3.2 \times 10^{-3} \\
q_{c} & =(20,2.5)^{T} \\
\operatorname{cov}(q) & =\left(\begin{array}{cc}
0.104 & -0.019 \\
-0.019 & 0.196
\end{array}\right) .
\end{array}
$$

The constant $\alpha$ is a fixed scaling factor. Opposite to the previous example, the direction of maximal variation of the model varies. A finite error of the UNG method will thus be obtained. To enable a numerical comparison to the LIN method, $q_{c}$ is displaced from the origin. The accuracy of the UNG method was determined by MC simulations with $10^{7}$ realizations, see Table 1. Its uncertainty was estimated from the typical variation for repeated simulations and transferred to the result of the UNG and LIN methods. 
The correct scent $\zeta=33 \%$ is given by the negative error of estimating $h_{c}$ with the LIN method. It is large, as expected from the asymmetry of the strongly nonlinear model. The nonlinear shift of the half-width $h_{p}$ is similarly obtained as the negative error for LIN, $\Delta h_{p}=4 \%$. Expanding the model to a higher order only to improve the calculation of $h_{p}$ would be inconsistent and hardly improve the result. The accuracy of the UNG method is high: There is essentially no error of either $h_{c}$ nor the halfwidth $h_{p}$ of the confidence interval. The confidence domain is illustrated in Fig. 3. The estimated and true (shown) confidence domains were indistinguishable.

Methods to test the validity of UNG without reference methods were derived in Section 4.3. The result of applying them here is presented in Table 2 . The directional error $\phi$ is less than 1 degree so the estimates are accurate. As this applies for both UNG directions $\tilde{d}=s \tilde{\nabla} h\left(q_{c}\right)$, the confidence boundaries are very close to be parallel. The magnitude

TABLE 1: Estimates of the confidence interval for the static example 2 (Section 6.2). The errors are relative to the half-width $h_{p}=1.078$ for the MC simulation. For repeated MC simulations, the limits $\left[h_{c}-h_{p}, h_{c}+h_{p}\right]$ typically varied $\pm 0.2 \%$ of $h_{p}$

\begin{tabular}{|c|c|c|c|c|}
\hline Method & {$\left[h_{c} \pm h_{p}\right]$} & {$\left[h_{c}-h_{p}, h_{c}+h_{p}\right]$} & Error $(\%)$ & Scent error $\delta \zeta(\%)$ \\
\hline MC / RSM(3) & {$[1.358 \pm 1.078]$} & {$[0.280,2.437]$} & {$[0,0]$} & 0 \\
\hline LIN & {$[1.000 \pm 1.038]$} & {$[-0.038,2.038]$} & {$[-29,-37]$} & -33 \\
\hline LIR / RSM(1) & {$[1.185 \pm 1.172]$} & {$[0.014,2.357]$} & {$[-12,-4]$} & -8 \\
\hline RSM(2) & {$[1.343 \pm 1.054]$} & {$[0.289,2.396]$} & {$[1,-4]$} & -1 \\
\hline UNG & {$[1.360 \pm 1.080]$} & {$[0.280,2.440]$} & {$[0.1,0.3]$} & 0.2 \\
\hline
\end{tabular}

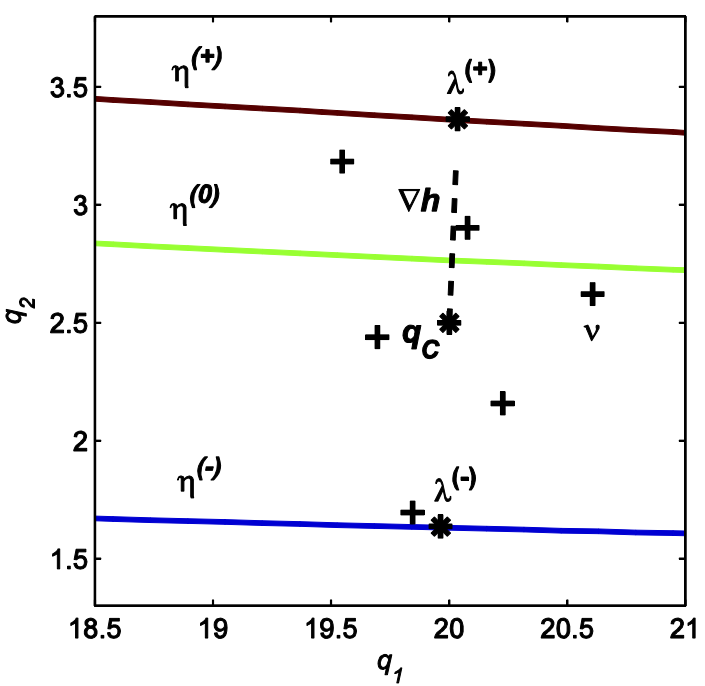

FIG. 3: Lambda points $\lambda^{( \pm)}$of the UNG method for the static example 2 (Section 6.2), constructed from the gradient $\nabla h$. The confidence boundaries $\eta^{( \pm)}$and the inverse centroid line $\eta^{(0)}$ indicating the scent are defined by, $h\left(\eta^{( \pm)}\right)=$ $h_{c} \pm h_{p}, h\left(\eta^{(0)}\right)=h_{c}$. The six points $v(+)$ provides a minimal set of samples for application of RSM(2), a second order RSM.

TABLE 2: Directional errors $(\phi)$ with resulting errors of the magnitude $\left(\delta \lambda_{\|}\right)$ of the lambda-point variations $\lambda-q_{c}$, and errors due to buckling $\left(\delta \lambda_{B}\right)$ of the confidence boundary $\eta$ for the static example 2 (Section 6.2)

\begin{tabular}{|c|c|c|c|}
\hline Method & $\phi \equiv \operatorname{acos}\left(\tilde{\nabla} h^{T} \tilde{d}\right)(\operatorname{deg})$ & $\delta \lambda_{\|} /\left|\lambda-q_{c}\right|(\%)$ & $\delta \lambda_{B} /\left|\lambda-q_{c}\right|(\%)$ \\
\hline UNG & {$[0.8,0.8]$} & {$[0.3,0.3]$} & {$[0.0,0.0]$} \\
\hline
\end{tabular}


error $\delta \lambda_{\|}$appears to be consistent with Fig. 3, even though the precision ( $\left.0.2 \%\right)$ of the MC simulation is too low to resolve the discrepancy. The minute buckling error $\delta \lambda_{B}$ clearly indicates why the planar approximation of the confidence boundary works well. In Fig. 3 it is indeed difficult to discern any curvature at all that would render a buckling error.

\subsection{Static Model 3}

An example of a static model which motivates higher order UN methods than the UNG is

$$
\begin{aligned}
h & =\alpha\left(q_{1}^{3}-q_{2}^{3}\right), \quad \alpha=4 \times 10^{-2} \\
q_{c} & =(1,1)^{T} \\
\operatorname{cov}(q) & =\left(\begin{array}{ll}
1.962 & 0.192 \\
0.192 & 1.038
\end{array}\right) .
\end{aligned}
$$

The covariance is very large to illustrate the breakdown of the UNG method. The direction of the gradient of the model changes considerably over the large confidence domain $\Omega$. It results in a substantial error of the UNG method, see Table 3. Also in this case, the MC simulation was made with $10^{7}$ samples. In Fig. 4, the confidence boundaries $\eta^{( \pm)}$are shown and the lambda points of the UNG and UNR methods are compared.

TABLE 3: Estimates of the confidence interval for the static example 3 (Section 6.3)

\begin{tabular}{|c|c|c|c|c|}
\hline Method & {$\left[h_{c} \pm h_{p}\right]$} & {$\left[h_{c}-h_{p}, h_{c}+h_{p}\right]$} & Error $(\%)$ & Scent error $\delta \zeta(\%)$ \\
\hline MC & {$[0.479 \pm 1.444]$} & {$[-0.965,1.922]$} & {$[0,0]$} & 0 \\
\hline LIN & {$[0 \pm 0.380]$} & {$[-0.380,0.380]$} & {$[41,-107]$} & -33 \\
\hline RSM(1) & {$[-0.035 \pm 0.862]$} & {$[-0.896,0.827]$} & {$[2,-38]$} & -18 \\
\hline RSM(2) & {$[0.626 \pm 1.356]$} & {$[-0.730,1.982]$} & {$[16,4]$} & 10 \\
\hline UNG & {$[0 \pm 0.699]$} & {$[-0.699,0.699]$} & {$[18,-85]$} & -33 \\
\hline UNR & {$[0.546 \pm 1.485]$} & {$[-0.939,2.032]$} & {$[2,8]$} & 5 \\
\hline
\end{tabular}

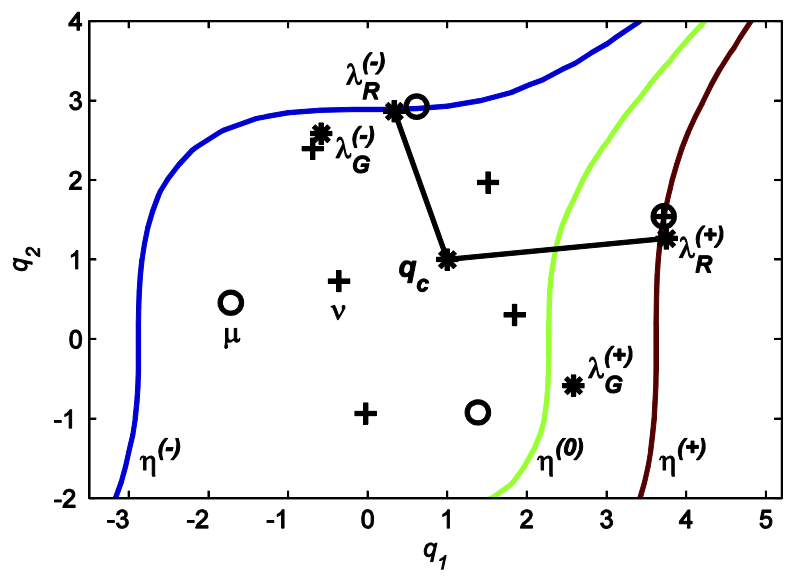

FIG. 4: Lambda points $\lambda_{R}^{( \pm)}$of the UNR method (Section 4.2) and the corresponding points $\lambda_{G}^{( \pm)}$of the UNG method (Section 4.1). The confidence boundaries $\eta^{( \pm)}$and the line $\eta^{(0)}$ indicating the scent are defined by $h\left(\eta^{( \pm)}\right)=h_{c} \pm$ $h_{p}, h\left(\eta^{(0)}\right)=h_{c}$. The four mu samples $\mu(\circ)$ are used for determining the directions from the center $q_{c}$ to the two lambda-points $\lambda_{R}^{( \pm)}$. The six points $v(+)$ provides a minimal set of samples for application of RSM(2), a second-order RSM. 
The UNG method mainly fails to estimate the upper confidence limit due to the very large variation of the direction of the gradient. The UNG method incorrectly claims $\zeta=0$. Using different directions to estimate the upper and lower confidence limits as in the UNR method results in a substantial improvement. Considering the large covariance, the estimated scent $\zeta=0.546$ for UNR is quite close to the correct value $\zeta=0.479$. Even though the directions $d^{( \pm)}$ from $q_{c}$ to $\lambda_{R}^{( \pm)}$do not cross the proper confidence boundaries $\eta^{(+)}$and $\eta^{(-)}$orthogonally, they nevertheless hit these boundaries fairly well. Consequently, the error of the estimated confidence interval of the model is acceptable for the UNR method, and much less than for the UNG method. This illustrates how the UNG method might fail and that it is possible to construct higher order methods with better performance. Finally, note that the computational cost is almost identical for the UNG and UNR methods. Only seven evaluations of the models are made with the UNR method, and six with the UNG method if the gradient is evaluated with a secant approximation [13].

Further comparison is provided here by application of RSM as well as a variant of LIN based on regression, denoted LIR. The $\operatorname{RSM}(n)$ calculation of order $n$ here utilizes surrogate models with mixed polynomials of order $n$. Details are given in Appendix A. Compared to the Gauss approximation formula, LIR offers an additional constant offset of $h_{c}$ from $h\left(q_{c}\right)$. This additional degree of freedom allows for an estimate of the scent. The linear model is identical to RSM(1) while the confidence interval is evaluated as in LIN, see Appendix B.

Methods to test the validity of UNG and UNR without reference methods were derived in Section 4.3. The result of applying them here is presented in Table 4. In contrast to example 2 in Section 6.2, the directional errors $\phi$ are substantial, so the accuracy of the error estimates may be questionable. Based on the large misalignments of $\lambda$ of the $\mathrm{UNG}$, it is fair to state that it fails in this example. For the UNR, $\phi$ may be regarded acceptable as the relevant scalar products $\tilde{d}^{T} \tilde{\nabla} h$ will only be $[-5 \%,-2 \%]$ off. Even though the UNR method may be accurate for this reason, it does not imply that the error estimates are equally good. The gradient as well as the Hessians can differ substantially since the estimated and ideal $\lambda^{(s)}$ are well separated in $Q$. This is difficult to account for in any other way than iteratively reevaluating $\lambda^{(s)}$ with directions $\tilde{d}^{(s)}$ better aligned with $\tilde{\nabla} h\left(\lambda^{(s)}\right)$. Indeed, in Fig. $4 \lambda^{(s)}$ appears to be close to inflection points with a large variation of the Hessian, especially $\lambda^{(-)}$. That could make the quadratic expansion inaccurate in Eq. (19). Comparing with Fig. 4, we find the error estimates give a fair but not accurate (in particular $\delta \lambda_{B}$ ) estimate of how far the points $\lambda^{(s)}$ are from the true confidence boundary $\eta^{(s)}$. The error estimates are thus not good for correction but detect failures qualitatively correct.

\subsection{Dynamic Correction of Measurement}

High-voltage voltage dividers are widely used in high-voltage measurements and testing in laboratories. Voltage dividers are required to reduce high voltages to measurable levels. The lightning test to be analyzed here is rather fierce for many components and important in the development of safe electrical high-voltage equipment. The typical response times of common voltage dividers are comparable to the rise time of lightning. The measurement is thus dynamic [20] and consequently needs to be analyzed as such [23]. The task considered here is to correct the measured signal with a digital correction filter [21] and associate a time-dependent uncertainty to the corrected signal [22].

\subsubsection{The Measurement and Its Correction}

Neglecting the (high) load impedance of the measurement equipment attached to the low-voltage connection, the equivalent circuit of the voltage divider is shown in Fig. 5. The parameters given in Table 5 were chosen to reflect

TABLE 4: Directional errors $(\phi)$ with resulting errors of the magnitude $\left(\delta \lambda_{\|}\right)$ of the lambda-point variations $\lambda-q_{c}$, and errors due to buckling $\left(\delta \lambda_{B}\right)$ of the confidence boundary $\eta$ for the static example 3 (Section 6.3)

\begin{tabular}{|c|c|c|c|}
\hline Method & $\phi \equiv \operatorname{acos}\left(\tilde{\nabla} h^{T} \tilde{d}\right)(\mathrm{deg})$ & $\delta \lambda_{\|} /\left|\lambda-q_{c}\right|(\%)$ & $\delta \lambda_{B} /\left|\lambda-q_{c}\right|(\%)$ \\
\hline UNG & {$[42,42]$} & {$[4.9,-49.9]$} & {$[-2.6,-1.4]$} \\
\hline UNR & {$[19,12]$} & {$[6.5,2.1]$} & {$[1.3,1.1]$} \\
\hline
\end{tabular}




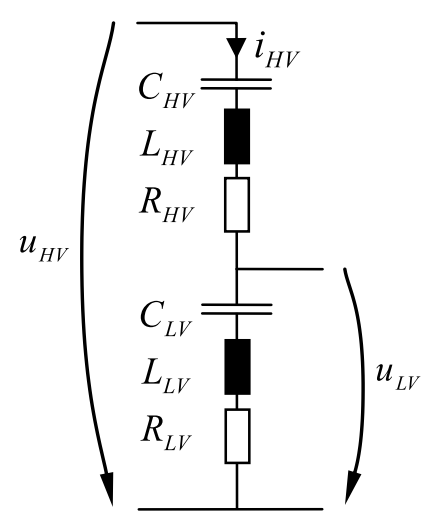

FIG. 5: Equivalent electrical circuit diagram of a high voltage voltage divider (Section 6.4), with resistive $R$, inductive $L$, and capacitive $C$ parts. The high-voltage connection $u_{H V}$ to the device under test is to the left, while the low-voltage connection $u_{L V}$ to the high-impedance voltmeter is to the right.

TABLE 5: Nominal resonance parameters of voltage divider. The nominal resistance $R_{c}$, inductance $L_{c}$, and capacitance $C_{c}$ are related as, $L_{c} C_{c}=1 / 4 \pi^{2} \phi^{2}, R_{c} C_{c}=\xi / \pi \phi$

\begin{tabular}{|c|c|c|}
\hline$c_{0}$ & $1 / 1000$ & Nominal ratio of voltage division \\
\hline$\left[\phi_{H V}, \phi_{L V}\right]$ & {$[2.3,0.8]$} & Resonance freq. (MHz) HV, LV circuits \\
\hline$\left[\xi_{H V}, \xi_{L V}\right]$ & {$[1.2,0.4]$} & Relative damping HV, LV circuits \\
\hline
\end{tabular}

common voltage dividers [24], rather than identified [25, 26] from calibration measurements, or calculated from first principles.

The dynamic model, or transfer function $F(s)$ of the voltage divider, is directly found by means of the standard rule of voltage division with impedances expressed in their Laplace transform,

$$
\begin{aligned}
F\left(q=c_{0}^{-1}, a_{1}, a_{2}, b_{1}, b_{2}, s\right)=c_{0} & \frac{b_{1} s^{2}+b_{2} s+1}{a_{1} s^{2}+a_{2} s+1}, c_{0} \equiv \frac{1}{1+C_{L V} / C_{H V}} \ll 1, \\
& \left\{\begin{array}{l}
b_{1} \equiv L_{L V} C_{L V} \\
b_{2} \equiv R_{L V} C_{L V}
\end{array},\left\{\begin{array}{l}
a_{1} \equiv\left(L_{H V}+L_{L V}\right)\left(C_{H V}^{-1}+C_{L V}^{-1}\right)^{-1} \\
a_{2} \equiv\left(R_{H V}+R_{L V}\right)\left(C_{H V}^{-1}+C_{L V}^{-1}\right)^{-1}
\end{array}\right.\right.
\end{aligned}
$$

The correction is given by the regularized approximation of the inverse transfer function, $\hat{F}^{-1}(s) \approx 1 / F(s) \equiv H(s)$. Since the model $F$ is independent of the current $i_{H V}$ (see Fig. 5), the prototype $1 / F(s)$ for the correction has five uncertain parameters even though there are six electrical elements of the voltage divider. The sampling rate was set to $50 \mathrm{MHz}$. Simplifications were made to focus on the propagation of uncertainty of the correction: The simple exponential mapping of poles and zeros was utilized to sample the continuous time correction $H(s)$ into the digital filter $g(q, t)$. Explicit regularization with low-pass noise filtering [21] was excluded since that was not required with the simplified model [23]. The filter coefficients $q=c_{0}^{-1}, a_{1}, a_{2}, b_{1}, b_{2}$ were linearized in electrical parameters $\rho=R_{H V}, L_{H V}, C_{H V}, R_{L V}, L_{L V}, C_{L V}$ to focus on the nonlinear dynamic mapping from $q$ to $H(q, s)$ and to disregard any additional nonlinear static mapping from $\rho$ to $q$.

The measurable quantity of interest is a normalized standard lightning test pulse [27] $[\Theta(t)$ is the Heaviside step function],

$$
x(t)=\Theta(t)\left[\exp \left(-t / T_{F}\right)-\exp \left(-t / T_{R}\right)\right] / x_{\max }, \quad\left\{\begin{array}{l}
T_{R}=0.403 \mu s \\
T_{F}=71.3 \mu s
\end{array} .\right.
$$

The lambda points (vectors) $\lambda^{(s)}(t)$ depend on the coefficients $c_{0}^{-1}, a_{1}, a_{2}, b_{1}, b_{2}$ of the correction $H(s)$. These are positive, as they are given by products of $R, L$, and $C$. This constraint is controlled by choosing a uniform distribution. 
The covariance matrix $\operatorname{cov}(\rho)$ shown in Table 6 must be positive semi definite. This was verified, eig $[\operatorname{cov}(\rho)] \geq 0$. Note that even though the covariance $\operatorname{cov}(\rho)$ between different electrical parameters is small, $\operatorname{cov}(q)$ is large between different parameters of the correction and must not be neglected.

The measured signal and confidence interval for the correction, evaluated with MC simulations of $10^{6}$ realizations, are shown in Fig. 6.

Initially, the measured pulse $y(t)=f\left(q_{c}, t\right) * x(t)$ is well outside the confidence interval of the correction. The correction thus gives a distinctive improvement. After around $1 \mu$ s though, $y(t) \in\left[h_{c}-h_{p}, h_{c}+h_{p}\right]$ and the improvement with dynamic correction $g\left(q_{c}, t\right) * y(t)$ may be questioned. Obviously, dynamic correction does not always guarantee a better result. To decide if correction should be applied or not, accurate estimation of the dynamic uncertainty as addressed here is essential. In this strongly idealized simulation the correction of the simulated measurement is exact as it recovers the original signal without any error, $g\left(q_{c}, t\right) *\left[f\left(q_{c}, t\right) * x(t)\right] \equiv x(t)$ 一 the example thus demonstrates aspects of dynamic uncertainty propagation in the most transparent way.

\subsubsection{The LIN Method}

The LIN method described in Section 5 relies upon the gradient signals for the correction and assigment of a universal approximation of the coverage factor signal, shown in Fig. 7.

TABLE 6: The relative covariance matrix for electrical parameters, $\rho=R_{H V}, L_{H V}, C_{H V}$, $R_{L V}, L_{L V}, C_{L V}$, linearly transformed to the relative covariance of the coefficients of correction $q=c_{0}^{-1}, a_{1}, a_{2}, b_{1}, b_{2}$

\begin{tabular}{|c|c|cc|}
\hline $\operatorname{cov}\left(\left\{\rho_{k} /\left(\rho_{c}\right)_{k}\right\}\right)$ & $\operatorname{cov}\left(\left\{q_{k} /\left(q_{c}\right)_{k}\right\}\right)$ \\
\hline $10^{-4}$ & $\left(\begin{array}{cccccc}6^{2} & 4^{2} & 0 & 0 & 0 & 0 \\
4^{2} & 7^{2} & 0 & 0 & 0 & 0 \\
0 & 0 & 1^{2} & 0 & 0 & 0 \\
0 & 0 & 0 & 7^{2} & 5^{2} & 0 \\
0 & 0 & 0 & 5^{2} & 9^{2} & 0 \\
0 & 0 & 0 & 0 & 0 & 2^{2}\end{array}\right)$ & $10^{-4}\left(\begin{array}{ccccc}5.0 & -1.0 & -1.0 & 6.2 & 4.0 \\
-1.0 & 49 & 17 & 1.1 & 0.2 \\
-1.0 & 17 & 37 & 0.0 & 0.1 \\
6.2 & 1.1 & 0.0 & 208 & 45 \\
4.0 & 0.2 & 0.1 & 45 & 53\end{array}\right)$ \\
\hline
\end{tabular}

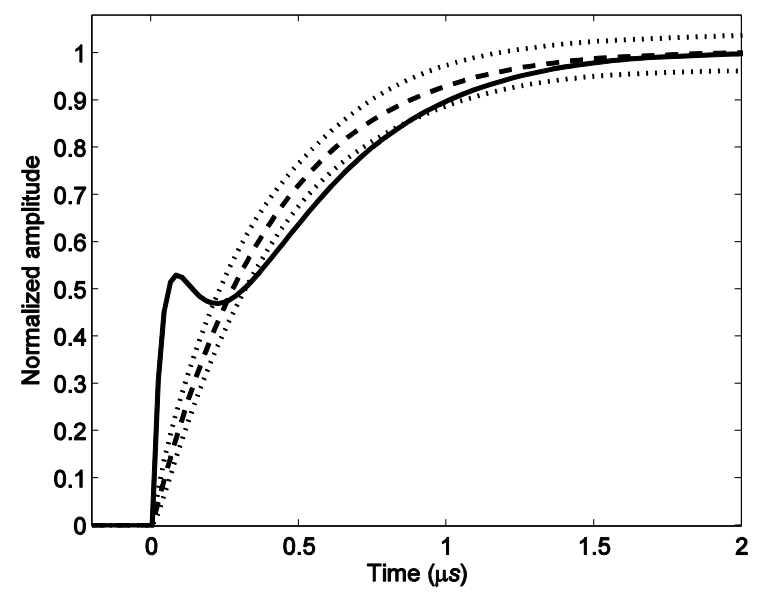

FIG. 6: Results of applying the reference MC method in the dynamic example (Section 6.4): The measured impulse $y(t)=f\left(q_{c}, t\right) * x(t)$ (solid) and the estimated confidence interval limits $\left[h_{c}(t)-h_{p}(t), h_{c}(t)+h_{p}(t)\right]$ (dotted) and center $h_{c}(t)$ (dashed) of the dynamic correction $h(y, q, t)=g(q, t) * y(t)$, where $g(q, t)$ is the impulse response of the correction filter. Only the fast rising edge of the lightning impulse [Eq. (34)] is shown, as the other parts of the pulse contain minor dynamic effects. 


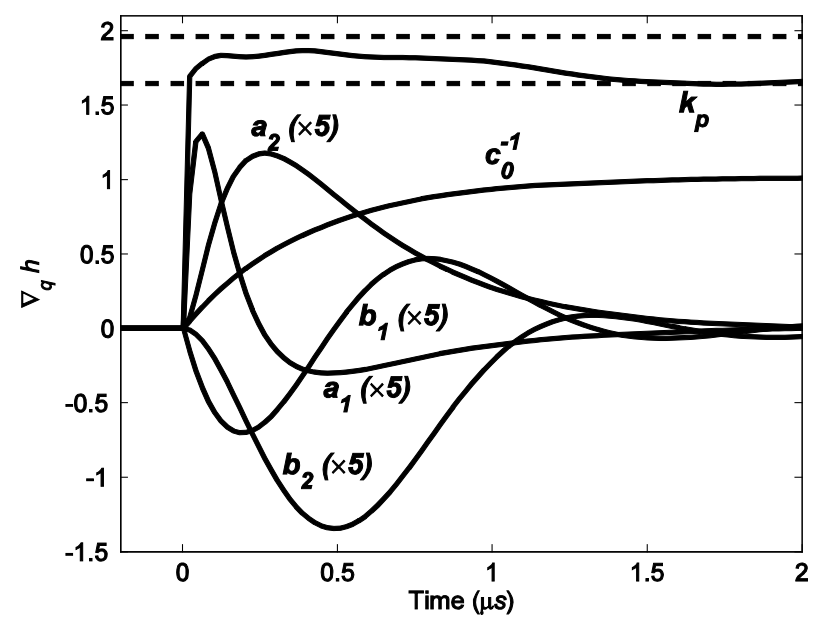

FIG. 7: The components $q=c_{0}^{-1}, a_{1}, a_{2}, b_{1}, b_{2}$ of the gradient $\nabla_{q} h(y, \bar{q}, t)$, for correction of the lightning impulse measured with the voltage divider. The time-dependent coverage factor $k_{p}$ is evaluated with the same MC simulations as used in Fig. 6. The limiting dashed lines at 1.645, 1.96 indicate coverage factors of uniform and normal distributions, respectively. In the static limit of constant signal, $k_{p} \rightarrow 1.645$, since then there is only one uniformly distributed static parameter $\left(c_{0}^{-1}\right)$.

The mere variation of the cover factor, $k_{p}(t) \in[1.640,1.866]$ results in an error $\left|\Delta h_{p}\right| / h_{p} \geq 7 \%$ of any approximate constant $k_{p}$. As stated in Section 2.2, the LIN method will always result in zero scent, $\zeta(t) \equiv 0$. Rather than more or less arbitrarily assign or guess a constant value of $k_{p}$ for LIN, which will grossly affect $h_{p}$, no further evaluation of LIN is made in this example. We conclude that it will give at least $7 \%$ relative error of the half-width $h_{p}$ in at least one instant of time (without additional compensating errors), and completely neglect the scent $\zeta(t)$ at all times. This sets the scale for comparing the proposed UN methods (UNG and UNR) with MC simulations.

\subsubsection{The UN Methods}

The lambda points $\lambda^{( \pm)}(t)$ were calculated according to Section 4.4 with $10^{6}$ random samples, using the covariance matrix $\operatorname{cov}(q)$ in Table 6 . For the UNG method, the matrix of gradient signals $\nabla_{q} h\left(y, q_{c}, t\right)$ was obtained with digital filtering as described in Section 5, and is shown in Fig. 7. For the UNR method, correction filters of all $\mu^{(s)}$ were realized and linear regression applied according to Section 4.2. The correction filters $g\left(\lambda^{(s)}(t), t\right)$ were then for both methods synthesized to filter the simulated measured lightning pulse $y(t)=f\left(q_{c}, t\right) * x(t)$, where $x(t)$ is given by Eq. (34), and $f\left(q_{c}, t\right)$ is the digital filter for $F\left(q_{c}, s\right)$ in Eq. (33). From the two resulting signals the corrections at the associated time instant $t$ were extracted to give the confidence interval limits according to Eq. (4). The resulting estimated scent $\zeta(t)=h_{c}(y, q, t)-h\left(y, q_{c}, t\right)$ and error of half-width $\Delta h_{p}(t)$ are displayed in Fig. 8. The accuracy of the UN methods were determined with Monte Carlo simulations. Acceptable accuracy required $10^{6}$ realizations.

Similarly to the static examples, the scent $\zeta$ strongly dominates nonlinear effects, as typical for nonlinear propagation of uncertainty (Section 2.2). The correct scent $\zeta(t)$ can be read off from the MC simulations (top, "MC"). Relative to the half-width $h_{p}$ it peaks around $10 \%$. The UNG method ("UNG") generally underestimates the scent and also fails in reproducing the oscillations after $0.5 \mu \mathrm{s}$. The UNR method ("UNR") is more accurate for the intervals with large scent. For $0.5 \mu \mathrm{s} \leq t \leq 1.0 \mu \mathrm{s}$ there is a noticable difference. Even if the error is not larger than about $2 \%$ of $h_{p}$, it illustrates inaccuracies of UNR. This happens when there is a substantial influence on the correction from many parameters, see the gradient signal in Fig. 7. The time-dependent confidence domain is then most complex and is extending in many of the possible directions of the five-dimensional parameter space. The half-width $h_{p}$ is accurately evaluated with both UN methods; the relative maximum error according to Fig. 8 (bottom) is about $1 \%$ of $h_{p}$. Initially though, the UNG method underestimates $h_{p}$ by about $2 \%$. After about $0.3 \mu$ s, the UNG method is more accurate 

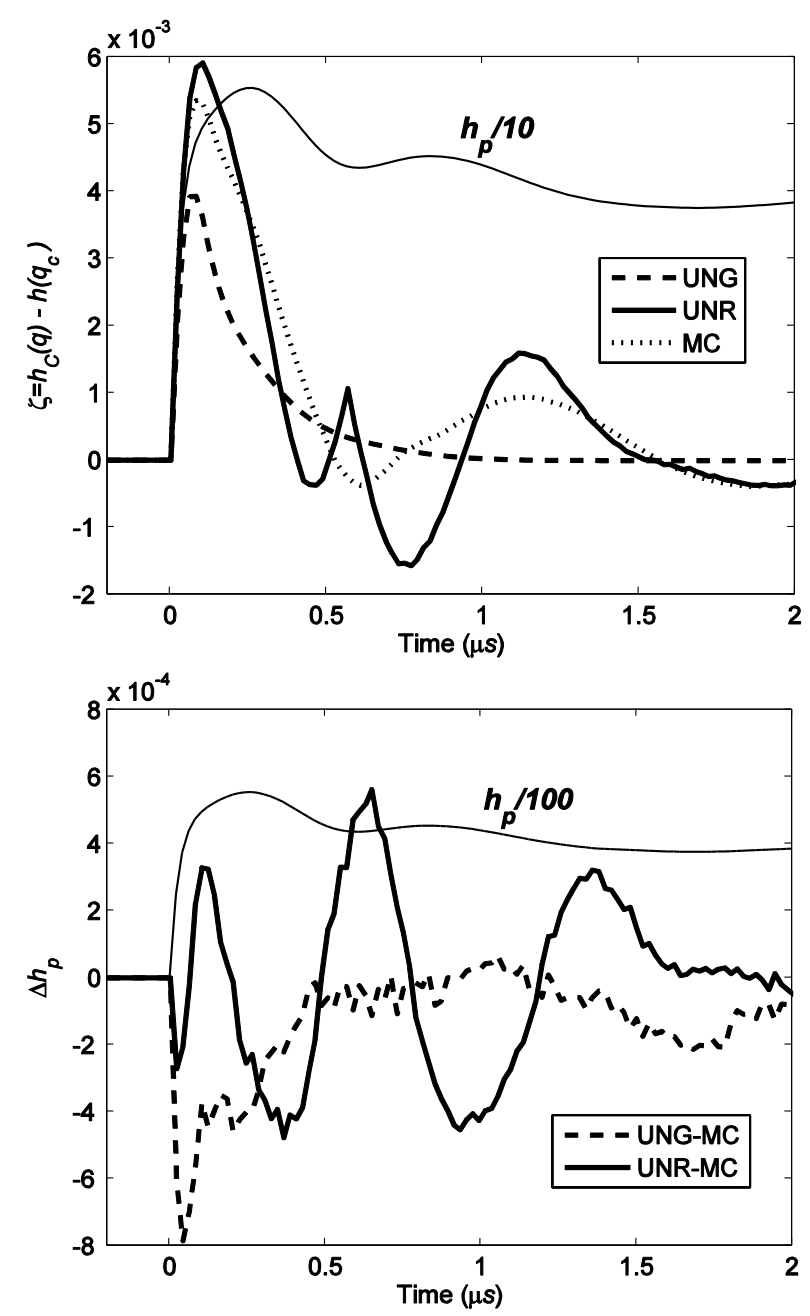

FIG. 8: Top: The estimated scent $\zeta(t)$ for the UNG, UNR, and MC (reference) methods. For LIN, $\zeta \equiv 0$. Bottom: The difference of calculated half-widths, for the UNG and UNR methods, relative MC. For LIN, $\Delta h_{p} / h_{p} \geq 7 \%$, see Section 6.4.2. The reduced half-widths $h_{p} / 10$ and $h_{p} / 100$ are included for comparison.

than UNR, which has an oscillating error. The error signals of the UNR method (top, bottom) are thus qualitatively similar. Overall, the error of the UNR method is slightly less than the UNG method. Their confidence intervals are nevertheless almost identical since the absolute errors are small. As anticipated, the errors are the largest where the correction is the strongest, see Fig. 6. Evaluating the performance, remember that confidence, or coverage intervals, almost without expectations are fairly approximate due to limited statistical knowledge.

\section{DISCUSSION}

The proposed UN approach has an apparent resemblance to RSM, which is described in Appendix A. In both cases, the model is first sampled in collocation points to find a model approximation. The subsequent steps are however distinctively different. RSM explores all possible variations of the parameters with a full MC simulation. The UN approach combines the potential variation of the approximate model with the actual variation of the parameters to determine the smallest possible set of points $\lambda^{(s)}$, at which the model should be sampled in a second stage. This 
maximum sparse sampling is made with the exact and not approximate model (as in RSM), to estimate the desired interval directly and not from a complete propagated pdf (as in RSM). The differences are substantial and result in different accuracy and computational efficiency for RSM and UN methods. The RSM errors relate to approximated sampling of the model, while all UN errors are due to approximate sampling of parameters. The efficiency defined by the minimal number of evaluations of the original model is given for some numbers of parameters and methods in Table 7 .

Clearly, the LIN and LIR methods are most efficient but also contain the coarsest approximations. The LIR (but not LIN) method roughly indicates the scent $\zeta$, as can been seen in examples in Sections 6.2 and 6.3. Estimating a nonlinear contribution to the result as the scent with a linear model may appear contradictory. It is possible since the linear model represents the mean, rather than a local (as in LIN) variation of the model, over a finite range of the parameters. If the distributions are normal, RSM(1) yields the same result as LIR, with the same efficiency. Increasing the order to RSM(2), the accuracy of the confidence intervals may improve substantially, see the examples in Sections 6.2 and 6.3. Unfortunately, the efficiency decreases rapidly with the number of parameters. An averagesized model with 20 parameters requires as many as 231 model evaluations to determine the surrogate model of RSM. For many finite element models, computational fluid dynamic, and dynamic models this may lead to unacceptable computation times. To improve the accuracy further, the order $r$ of the $\operatorname{RSM}(r)$ may be increased. If 100 model evaluations is the acceptable limit, at most $n=6$ uncertain parameters are allowed for $r=3, n=4$ for $r=4$, and not more than $n=3$ parameters for $r=5$. For moderate values of $r$ and large $n$, RSM should not be used at all since determining the surrogate model may require as many models evaluations as a brute force MC simulation. High-orders $r$ should generally be avoided due to the poor behavior of high- order polynomials.

The efficiency of the proposed UN methods scales linearly with the number of parameters. UNG requires $n+3$ model evaluations if the gradient $\nabla h\left(q_{c}\right)$ is estimated with a minimal number of points in the vicinity of $q_{c}$. To avoid using any sample (except $q_{c}$ ) more than once in UNR, at least $2 n+3$ samples are required. UNR thus has about half the efficiency of UNG, but is often considerably more accurate, as can be seen in the examples in in Sections 6.3 and 6.4. The main complications with the UN methods belong to two categories, one is related to accuracy and one to efficiency. Both the UNG and UNR method rely upon a (piecewise) constant direction of the gradient over the relevant part of the true confidence domain. Whenever this is violated (as in example in the Section 6.3), the accuracy is reduced. The other difficulty related to efficiency occurs for higher dimensional models $h \in \Re^{m}, m \gg 1$, or fields $h \in \Re^{m \times k}, m, k \gg 1$. The confidence domains will generally be different for different components $h_{m}$ and $\lambda^{(s)}$ should be estimated independently, as in the example in Section 6.4. This complication is shared with RSM, which also need unique surrogate models for all $h_{m}$. To obtain any result at all in this case, the high efficiency of UNG/UNR displayed in Table 7 may be crucial. There are also other variants of deterministic sampling which are more appropriate for vector and field models. Instead of focusing on varying confidence domains, it is more efficient to study quantities invariant over different components $h_{m}$, such as the statistical moments of the parameters. The two lambda points are then substituted with a larger set of samples which instead is universally $\left(\forall h_{m}\right)$ valid. This alternative was recently illustrated for a simple dynamic example [18] and will be further investigated in the future.

TABLE 7: Computational efficiency for $\operatorname{RSM}(n)$ [Eq. (A.3)] for selected orders $n$, and UNG/UNR

\begin{tabular}{|c|c|c|c|c|c|}
\hline $\begin{array}{c}\text { Parameters } \\
\text { method }\end{array}$ & 2 & 5 & 10 & 20 & 30 \\
\hline LIN/LIR/RSM(1) & 3 & 6 & 11 & 21 & 31 \\
\hline RSM(2) & 6 & 21 & 66 & 231 & 496 \\
\hline RSM(3) & 10 & 56 & 286 & 1,771 & 5,456 \\
\hline RSM(4) & 15 & 126 & 1001 & 10,626 & 46,376 \\
\hline RSM(5) & 21 & 252 & 3003 & 53,130 & 324,632 \\
\hline UNG & 5 & 8 & 13 & 23 & 33 \\
\hline UNR & 7 & 13 & 23 & 43 & 63 \\
\hline
\end{tabular}

Volume 3, Number 5, 2013 
By no means do the proposed UN methods exhaust all possible approximations. Higher order methods should account for the variation of the gradient direction. That includes rotation of the level hyperplanes of the model, as well as the buckling of each such hyperplane. By necessity, the complexity of the method will increase and the efficiency will decrease. The primary goal here is to propose a novel UN approach and illustrate that it can be efficient and accurate, rather than exploring all possible approximations.

\section{CONCLUSIONS}

A novel "UN" class of methods for nonlinear propagation of uncertainty by sampling at confidence boundaries has been introduced. The proposed UNG and UNR methods are two approximate methods of this class. The main limitation of these methods is their rudimentary topology of the estimated confidence domain. The main criterion for their validity is a low variation of the direction of maximal variation of the model in the parameter space, over the relevant range of parametric variation. That is analogous but different from the requirement of a low residual error of truncation in any finite Taylor expansion. The magnitude of model variation is irrelevant for the UNG and UNR methods, which makes them accurate and robust. For several reasons, the approach focuses on the complexity of the model equation, rather than the fidelity of statistical information (as is conventional):

1. Models are often more complex than probability distributions.

2. For confidence intervals, the dependence on statistical details is usually weak.

3. Statistical information is seldom known accurately.

More comparisons between the traditional and various approaches based on sampling on confidence boundaries are strongly desired, as well as refinements and more verification of the proposed methods for evaluating their validity.

\section{ACKNOWLEDGMENT}

Financial support for the National Metrology Program from VINNOVA (The Swedish Governmental Agency for Innovation Systems) is gratefully acknowledged.

\section{REFERENCES}

1. GUM, I., Guide to the Expression of Uncertainty in Measurement, Tech. Rep., International Organisation for Standardisation, Geneva, Switzerland, 1995.

2. Ramdani, N., Candau, Y., Guyon, G., and Dalibart, C., Sensitivity analysis of dynamic models to uncertainties in inputs data with time-varying variances, Technometrics, 48(1):74-87, 2006.

3. Metropolis, N. and Ulam, S., The Monte Carlo method, J. Am. Stat. Assoc., 44(247):335-341, 1949.

4. Metropolis, N., The Beginning of the Monte Carlo method, Los Alamos Sci. Special Issue, 15:125-130, 1987.

5. Wiener, N., The homogeneous chaos, Am. J. Math., 60:897-936, 1938.

6. Lovett, T., Polynomial Chaos Simulation of Analog and Mixed-Signal Systems: Theory, Modeling Method, Application, Lambert Academic Publishing, Saarbrücken, Germany, 2006.

7. Li, H. and Zhang, D., Probabilistic collocation method for flow in porous media: Comparisons with other stochastic methods, Water Resour. Res., 43:W09409, 2007.

8. Witteveen, J. A., Doostan, A., Chantrasmi, T., Pecnik, R., and Iaccarino, G., Comparison of stochastic collocation methods for uncertainty quantification of the transonic rae 2822 airfoil, In NODESIM-CFD Workshop on Quantification of CFD Uncertainties, Brussels, Belgium, Oct. 2009.

9. Helton, J. and Davis, F., Latin hypercube sampling and the propagation of uncertainty in analyses of complex systems, Reliab. Eng. Syst. Saf., 81:23-69, 2003.

10. Julier, S., Uhlmann, J., and Durrant-Whyte, H., A new approach for filtering non-linear systems, In American Control Conference, pp. 1628-1632, Seattle, Washington, 1995. 
11. Julier, S. and Uhlmann, J., Unscented filtering and nonlinear estimation, Proc. IEEE, 92(3):401-422, 2004.

12. Simon, D., Optimal State Estimation: Kalman, and Non-Linear Approaches, Wiley, Hoboken, NJ, 2006.

13. Svensson, T. and de Mare, J., On the choice of difference quotients for evaluating prediction intervals, Measurement, 41(7):755-62, 2008

14. Råde, L. and Westergren, B., Mathematics Handbook, Studentlitteratur, Lund, Sweden, 2nd ed., 1990.

15. Kalman, R., A new approach to linear filtering and prediction problems, ASME J. Basic Eng., 82:35-45, 1960.

16. Julier, S. and Uhlmann, J., The scaled unscented transformation, In Proceedings of the IEEE American Control Conference, pp. 4555-4559, Anchorage AK, USA, 2002.

17. Björk, Å., Numerical Methods for Least Squares Problems, SIAM, Philadelphia, 1996.

18. Hessling, J. P., Integration of digital filters and measurements, In Digital Filters, pp. 123-154, INTECH, Rijeka, Croatia, 2011.

19. Silva, D. S. and Skilling, J., Data Analysis: A Bayesian Tutorial, Oxford University Press, Oxford, 2006.

20. Hessling, J. P., Metrology for non-stationary dynamic measurements, In Advances in Measurement Systems, pp. 221-256, INTECH, Vukovar, Croatia, 2010.

21. Hessling, J. P., A novel method of dynamic correction in the time domain, Measur. Sci. Technol., 19:075101, 2008.

22. Hessling, J. P., A novel method of evaluating dynamic measurement uncertainty utilizing digital filters, Measur. Sci. Technol., 20:055106, 2009.

23. Hessling, J. P. and Mannikoff, A., Dynamic measurement uncertainty of HV voltage dividers, In XIX IMEKO World Congress, Lisbon, Portugal, 2009.

24. Bergman, A. and Hällström, J., Impulse dividers for dummies, In XIII International Symposium on High Voltage Engineerings, Netherlands, 2003.

25. Ljung, L., System Identification: Theory for the User, Prentice Hall, Englewood Cliffs, 2nd ed., 1999.

26. Pintelon, R. and Schoukens, J., System Identification: A Frequency Domain Approach, IEEE Press, Piscataway, NJ, 2001.

27. IEC, IEC 60060-2, 1994, High-Voltage Test Techniques Part 2: Measuring systems, Tech. Rep., International Electrotechnical Commission, Geneva, Switzerland, 1994.

28. Hombal, V. and Mahadevan, S., Bias minimization in gaussian process surrogate modeling for uncertainty quantification, Int. J. Uncertainty Quantif., 1(4):321-349, 2011.

\section{APPENDIX A. RESPONSE SURFACE METHODOLOGY WITH POLYNOMIAL SURROGATE MODELS}

A presumably complex model can be approximated with a model $h^{(R S M)}$ simple enough to perform a full MC simulation $[9,28]$. This simulation is then made as if the approximate model would be the true model. An $r$ th-order model approximation can be chosen as a mixed $r$ th-order polynomial,

$$
h \approx h^{(R S M(r))}=\sum_{k=0}^{r} \sum_{m=1}^{w(n, k)} a_{k m} \prod_{j=1}^{n} q_{j}^{e_{j k m}}: \begin{cases}\sum_{j=1}^{n} e_{j k m} & =k \\ e_{j k m} & \geq 0, \forall\{j, k, m\} .\end{cases}
$$

The number $w(n, k)$ of terms $a_{k m}$ can be found recursively,

$$
w(n, k)= \begin{cases}\sum_{j=1}^{\min (n, k)}\left(\begin{array}{c}
n \\
j
\end{array}\right) w(j, k-j), & k>0 \\
1, & k=0 .\end{cases}
$$

Unique determination of the surrogate model requires a full rank regression matrix. The true model must thus be sampled in at least $p$ collocation points $v=\left(\begin{array}{llll}v_{1} & v_{2} & \ldots & v_{p}\end{array}\right)$,

$$
p(r)=\sum_{k=0}^{r} w(n, k) .
$$

Volume 3, Number 5, 2013 
This number determines the efficiency of the RSM, provided the numerical cost for evaluating the true model dominates over the cost for the MC simulation of the surrogate model. The coefficients can be found using linear regression, similar to how the linear model parameters $h_{0}^{(s)}, d^{(s)}$ are determined from the collocation points $\mu$ of the UNR method [Section 4.2, Eqs. (7)-(9)]. A difference is that $h^{[R S M(r)]}$ is nonlinear for $r>1$. It may indeed be possible to use the same sets $\mu$ and $\nu$, but it may not be sufficient or optimal for RSM.

It is nontrivial to find optimal $v$ points which are evenly distributed in the most relevant region and are as few as possible for maximum computational efficiency. The matrix $V= \pm I$ for $\{\mu\}$ in Eq. (10) can be adapted to describe the primary variations of $\{v\}$,

$$
\begin{aligned}
\{v\} & =q_{c}+\left\langle k_{p}^{(s)}\right\rangle_{s} U^{T} \sqrt{U \operatorname{cov}(q) U^{T}} V, \\
V & =\left(\begin{array}{llll}
V^{(1)} & V^{(2)} & \ldots & V^{(n)}
\end{array}\right) .
\end{aligned}
$$

The variations are described by the columns of $V$, divided into submatrices $V^{(k)}$. The $v$ points contained in $V^{(k)}$ are here defined to have the same magnitude $\left\|V_{:, q}^{(k)}\right\|=\rho(k), \forall q$ and are thus contained within one "shell." The samples should be uniformly distributed within each shell $V^{(k)}$, with appropriate offset rotations between the shells. Selected $v$ points are preferably checked to be sufficient by verifying full rank of the regression matrix. This spherical geometry is appropriate since the scale $\sqrt{U \operatorname{cov}(q) U^{T}}$ has been extracted. If $\rho(k)=1, \forall k$, the regression matrix will be rank deficient for $r>1$ and $h^{[R S M(r)]}$ becomes ambiguous. The $\mu$ points chosen in Section 4.2 will thus not yield appropriate $v$ points for RSM if $r>1$. In the examples in Sections 6.2 and 6.3, $\rho(k)=\max (1, k) / r$ with $w(n, k) v$ points in the shell described by $V^{(k)}$ for $k>1$, but $w(n, 1)+1 \vee$ points for $k=1$.

\section{APPENDIX B. LINEARIZATION WITH REGRESSION (LIR)}

For RSM(1), MC simulations are not needed to propagate the covariance of the parameters. However, for non-normal probability distributions the evaluation of the coverage factor $k_{p}$ requires an MC simulation, as described in Section 4.4. The only difference between linearization with regression (LIR) and LIN [1] is one added degree of freedom describing an offset. Instead of blind assignment of the central point of the confidence interval of the modeling result to $h\left(q_{c}\right)$, it is an additional free parameter. In contrast to LIN, the LIR method gives a rough but not accurate indication of the scent. The confidence interval is given by

$$
\left[h_{c}^{(L I R)} \pm h_{p}^{(L I R)}\right]=\left(\begin{array}{cc}
1 & q_{c}^{T}
\end{array}\right)\left(\begin{array}{c}
a_{01} \\
a_{1:}
\end{array}\right) \pm k_{p}^{(s)} \sqrt{\left[a_{1:}\right]^{T} \operatorname{cov}(q) a_{1:}}, a_{1:} \equiv\left(\begin{array}{llll}
a_{11} & a_{12} & \cdots & a_{1 n}
\end{array}\right)^{T},
$$

where $h^{(R S M(1))}(q)=\left(\begin{array}{cc}1 & q^{T}\end{array}\right)\left(\begin{array}{c}a_{01} \\ a_{1:}\end{array}\right)$ is the linear surrogate model of RSM(1), see Appendix A. 